SFB

Tests based on simplicial depth for AR (1) models with explosion

Anne Leucht, Christoph P. Kustosz,

Christine $H$. Müller

$$
\text { Nr. 34/2014 }
$$
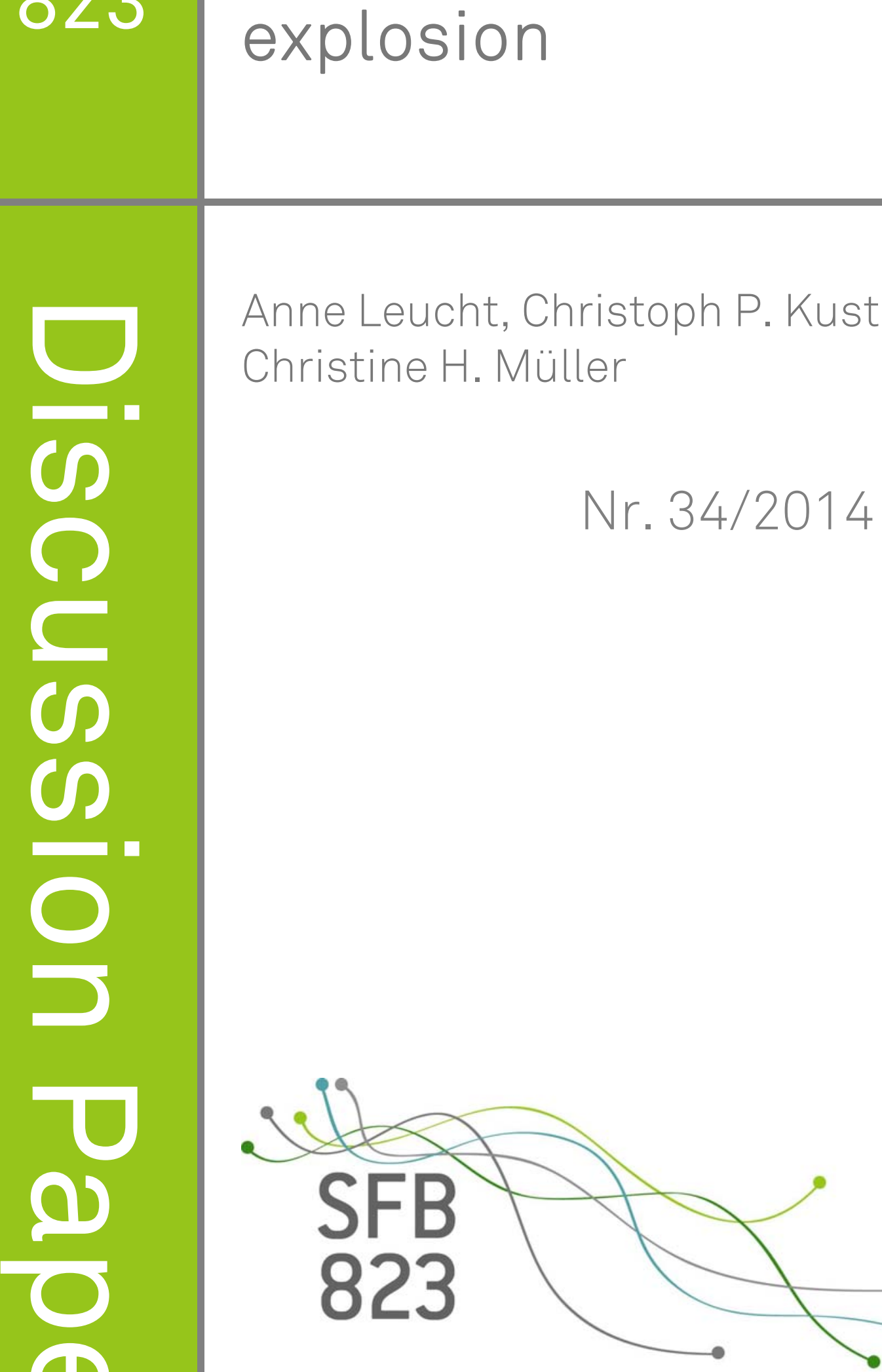



\title{
TESTS BASED ON SIMPLICIAL DEPTH FOR AR(1) MODELS WITH EXPLOSION
}

\author{
Anne Leucht \\ Technische Universität Braunschweig \\ Institut für Mathematische Stochastik \\ Pockelsstr. 14 \\ D-38106 Braunschweig \\ Germany \\ E-Mail: a.leucht@tu-bs.de \\ Christoph P. Kustosz and Christine H. Müller* \\ Technische Universität Dortmund \\ Vogelpothsweg 87 \\ D-44221 Dortmund \\ Germany \\ E-mail: kustosz@statistik.tu-dortmund.de, cmueller@statistik.tu-dortmund.de
}

\begin{abstract}
We propose an outlier robust and distributions-free test for the explosive AR(1) model with intercept based on simplicial depth. In this model, simplicial depth reduces to counting the cases where three residuals have alternating signs. Using this, it is shown that the asymptotic distribution of the test statistic is given by an integrated two-dimensional Gaussian process. Conditions for the consistency of the test are given and the power of the test at finite samples is compared with five alternative tests, using errors with normal distribution, contaminated normal distribution, and Fréchet distribution in a simulation study. The comparisons show that the new test outperforms all other tests in the case of skewed errors and outliers. Although here we deal with the AR(1) model with intercept only, the asymptotic results hold for any simplicial depth which reduces to alternating signs of three residuals.
\end{abstract}

2000 Mathematics Subject Classification. Primary 62M10, 62G10; secondary 62G35, $62 \mathrm{~F} 05$.

Keywords. Alternating signs, autoregression, explosion, distribution-free test, robustness, simplicial depth, asymptotic distribution, consistency, power comparison. Short title. Simplicial depth for AR(1) models.

version: October 7, 2014

${ }^{*}$ Research supported by DFG via the Collaborative Research Centre SFB 823 'Statistical modelling of nonlinear dynamic processes' (Project B5). 


\section{INTRODUCTION}

Consider the $\mathrm{AR}(1)$ model

$$
Y_{n}=\theta_{0}+\theta_{1} Y_{n-1}+E_{n}, \quad n=1, \ldots, N,
$$

where $E_{1}, \ldots, E_{N}$ are i.i.d. errors with $\operatorname{med}\left(E_{n}\right)=0, P\left(E_{n}=0\right)=0$, and $Y_{0}=y_{0}$ is the starting value. Moreover, it is assumed that $Y_{n}$ is almost surely strictly increasing, so that $Y_{n}$ is an exploding process and $\theta_{1}>1$. An example of such an exploding process is crack growth, where a stochastic version of the Paris-Erdogan equation provides $Y_{n}=\theta_{1} Y_{n-1}+\widetilde{E}_{n}$, whereby $\widetilde{E}_{n}$ is nonnegative, see Kustosz and Müller (2014a). Setting $\theta_{0}=\operatorname{med}\left(\widetilde{E}_{n}\right)$ and $E_{n}=\widetilde{E}_{n}-\theta_{0}$, we obtain model 1.1 ) for this case. The aim is to test a hypothesis $H_{0}: \theta=\left(\theta_{0}, \theta_{1}\right)^{\top} \in \Theta_{0}$, where $\Theta_{0}$ is a subset of $[0, \infty) \times(1, \infty)$. In particular, the aim is to test hypotheses on the median of the distribution of $\widetilde{E}_{n}$ as well.

While there is a vast literature on stationary $\operatorname{AR}(1)$ models with $\left|\theta_{1}\right|<1$ and for the unit root case with $\theta_{1}=1$, there exist only few results for the explosive case. Anderson (1959) derived the asymptotic distribution of estimators when the errors $E_{n}$ are assumed to be independent and normally distributed. Basawa et al. (1989) and Stute and Gründer (1993) used bootstrapping methods to derive the asymptotic distribution of estimators and predictors without assuming the normal distribution. Special maximum likelihood estimators for $\mathrm{AR}(1)$ processes with nonnormal errors were treated by Paulaauskas and Rachev (2003). Recently Hwang and Basawa (2005), Hwang et al. (2007) and Hwang (2013) investigated the asymptotic distribution of the least squares estimator of explosive $\mathrm{AR}(1)$ processes under some modifications of the process like dependent errors $E_{n}$. Further the limit distribution of the Ordinary Least Squares estimator in case of explosive processes was examined by Wang and $\mathrm{Yu}$ (2013) and differs from the stationary case depending on the underlying error distribution.

The least squares estimator is known to be sensitive to innovation outliers and additive outliers as discussed in Fox (1972). Outlier robust methods for time series were mainly proposed recently, see e.g. Grossi und Riani (2002), Agostinelli (2003), Fried und Gather (2005), Maronna (2006), Grillenzoni (2009), Gelper et al. (2009). However these methods deal only with estimation and forecasting. An asymptotic distribution is not derived in these papers so that no tests can be used. Only Huggins (1989) proposed a sign test for stochastic processes based on a M-estimator. The robustness of the sign test was studied in Boldin (2011). Moreover Bazarova et al. (2014) derived the asymptotic distribution of trimmed sums for AR(1) processes. However all these approaches base heavily on the stationarity of the process so that they cannot be used for explosive processes.

Here we propose an outlier robust and distribution free test for hypotheses on $\theta=\left(\theta_{0}, \theta_{1}\right)^{\top}$ for the explosive $\mathrm{AR}(1)$ model given by 1.1$)$ which is based on simplicial depth. Simplicial depth was originally introduced by Liu $(1988,1990)$ to provide another generalization of the outlier robust median to multivariate data. The direct generalization of the median to multivariate data is the half-space depth proposed by Tukey (1975). In this definition, the depth of a $p$-dimensional parameter $\mu$ within 
a $p$-dimensional data set is the minimum relative number of data points lying in a half-space containing the parameter $\mu$. If there are $p+1$ data points then they span a $p$-dimensional simplex and all points inside the simplex have the half-space depth of $1 /(p+1)$ and all points outside the simplex have the depth 0 . The simplicial depth of Liu defines the depth of a parameter $\mu$ as the relative number of simplices spanned by $p+1$ data points which contain the paramter $\mu$, i.e. where the half-space depth of $\mu$ with respect to the $p+1$ data points is greater than 0 . Replacing the halfspace depth by other depth notions leads to a corresponding simplicial depth. For example, Rousseeuw and Hubert (1999) generalized the half-space depth to regression by introducing the concept of nonfit. Thereby the depth of a regression function or respectively the regression parameter $\theta$ within a data set is the minimum relative number of data points which must be removed so that the regression function becomes a nonfit. The corresponding simplicial regression depth of a $p$-dimensional parameter $\theta$ is then the relative number of subsets with $p+1$ data points so that $\theta$ is not a nonfit with respect to these $p+1$ data points, see Müller (2005). Mizera (2002) proposed a general depth notion by introducing a quality function. Usually the quality function is given by the residuals. Here the residuals of the $\operatorname{AR}(1)$ process are used as well.

Simplicial depth has the advantage that it is a U-statistic so that its asymptotic distribution is known in principle. This is usually not the case for the original depth notion. However, the simplicial depth often is a degenerated U-statistic. There are only few cases where this is not the case, see Denecke and Müller (2011, 2012, 2013, 2014). For regression problems, the simplicial depth is a degenerated U-statistic. Deriving the spectral decomposition of the conditional expectation, Müller (2005), Wellmann et al. (2009) and Wellmann and Müller (2010a,b) derived the asymptotic distribution for several regression problems with independent observations. Only the most simple case, namely linear regression through the origin, can be transferred to $\mathrm{AR}(1)$ regression with no intercept $\theta_{0}$. In this case, the asymptotic distribution is given by one $\chi^{2}$-distributed random variable. This was done in Kustosz and Müller (2014a). However, as soon as more than one parameter is unknown, the approaches for an asymptotic distribution developed for regression with independent observations cannot be transferred to autoregression. For example for polynomial regression with independent observations, the asymptotic distribution is given by an infinite sum of $\chi^{2}$-distributed random variables. Here we show, that the asymptotic distribution for the $\mathrm{AR}(1)$ model is given by an integrated two-dimensional Gaussian process. Crucial for this result is that simplicial depth in this model reduces to counting the subsets of three data points where the residuals have alternating signs. Therefore this asymptotic distribution does not hold only for AR(1) models with intercept but also for other models where simplicial depth is given by the number of alternating signs of three residuals. For example it also holds for the nonlinear AR(1) model $Y_{n}=\theta_{1} Y_{n-1}^{\theta_{2}}+E_{n}$ under similar assumptions as stated above.

In Section 2, we provide the simplicial depth for the $\mathrm{AR}(1)$ model with intercept given by (1.1) and the test statistics based on this simplicial depth for hypotheses about $\theta=\left(\theta_{0}, \theta_{1}\right)^{\top}$. To obtain the critical values of the tests, the asymptotic distribution of the simplicial depth is derived in Section 3. In particular, this asymptotic distribution does not depend on the starting value $y_{0}$ of the process as it is the case for the asymptotic distribution of the least squares estimator, see e.g. Hwang (2013). In Section 4, we derive the consistency of the tests given in Section 2. Although the 
test statistic is given by a very simple form, the calculation of it could be lengthy since all subsets of three observations have to be considered. In Section 5 an efficient algorithm for its calculation is shortly described as well as the efficient calculation of quantiles of the asymptotic distribution. Using this, a power simulation of the new test is given and the power is compared with the power of five other tests, where three of them are based on a simplified version of the depth notion given in Kustosz and Müller (2014b).

\section{Simplicial DEPTH FOR The AR(1) MODEL}

According to Mizera (2002), we need a quality function to define a depth notion for the AR(1) model (1.1). A natural quality function is the function given by the squared residuals. Set $\theta=\left(\theta_{0}, \theta_{1}\right)^{\top}$ and define the residuals by

$$
r_{n}(\theta)=y_{n}-\theta_{0}-\theta_{1} y_{n-1}, n=1, \ldots, N,
$$

where $y_{n}$ is the realization of $Y_{n}$ for $n=0, \ldots, N$.

Tangential depth of the parameter $\theta$ in the sample $y_{*}=\left(y_{0}, \ldots, y_{N}\right)^{\top}$ is then (see Mizera 2002)

$$
d_{T}\left(\theta, y_{*}\right)=\frac{1}{N} \min _{|u|=1} \sharp\left\{n \in\{1, \ldots, N\} ; u^{\top} \frac{\partial}{\partial \theta} r_{n}(\theta)^{2} \leq 0\right\}
$$

so that it becomes

$$
d_{T}\left(\theta, y_{*}\right)=\frac{1}{N} \min _{|u|=1} \sharp\left\{n \in\{1, \ldots, N\} ; r_{n}(\theta) u^{\top}\left(\begin{array}{c}
1 \\
y_{n-1}
\end{array}\right) \leq 0\right\}
$$

here. To define simplicial depth for autoregression, it is useful to write the sample in pairs, i.e. the sample is given by $z_{*}=\left(z_{1}, \ldots, z_{N}\right)^{\top}$ where $z_{n}=\left(y_{n}, y_{n-1}\right)^{\top}$. Then, simplicial depth of a p-dimensional parameter $\theta \in \mathbb{R}^{p}$ in the sample $z_{*}$ is in general (see Müller 2005)

$$
d_{S}\left(\theta, z_{*}\right)=\frac{1}{\left(\begin{array}{c}
N \\
p+1
\end{array}\right)} \sum_{1 \leq n_{1}<n_{2}<\ldots<n_{p+1} \leq N} \mathbb{1}\left\{d_{T}\left(\theta,\left(z_{n_{1}}, \ldots, z_{n_{p+1}}\right)\right)>0\right\},
$$

where $\mathbb{1}\left\{d_{T}\left(\theta,\left(z_{1}, \ldots, z_{p+1}\right)\right)>0\right\}$ denotes the indicator function $\mathbb{1}_{A}\left(z_{1}, \ldots, z_{p+1}\right)$ with $A=\left\{\left(\bar{z}_{1}, \ldots, \bar{z}_{p+1}\right)^{\top} \in \mathbb{R}^{p+1}\right.$ $\left.d_{T}\left(\theta,\left(\bar{z}_{1}, \ldots, \bar{z}_{p+1}\right)\right)>0\right\}$. The simplicial depth is a U-statistic. In the $\mathrm{AR}(1)$ model (1.1), it becomes

$$
d_{S}\left(\theta, z_{*}\right)=\frac{1}{\left(\begin{array}{c}
N \\
3
\end{array}\right)} \sum_{1 \leq n_{1}<n_{2}<n_{3} \leq N} \mathbb{1}\left\{d_{T}\left(\theta,\left(z_{n_{1}}, z_{n_{2}}, z_{n_{3}}\right)\right)>0\right\} .
$$

If the regressors $y_{n-1}$ satisfy $y_{n_{1}-1}<y_{n_{2}-1}<y_{n_{3}-1}$ for $n_{1}<n_{2}<n_{3}$, then $d_{T}\left(\theta,\left(z_{n_{1}}, z_{n_{2}}, z_{n_{3}}\right)\right)>0$ if and only if the residuals $r_{n_{1}}, r_{n_{2}}, r_{n_{3}}$ have alternating signs or at least one of them is zero (see Kustosz and Müller 2014b). Since $Y_{n}$ is almost surely strictly increasing by assumption, we can always assume $y_{n_{1}-1}<y_{n_{2}-1}<y_{n_{3}-1}$ 
for $n_{1}<n_{2}<n_{3}$ without loss of generality. Moreover, since $P\left(E_{n}=0\right)=0$, we can restrict ourselve to residuals with alternating signs so that almost surely

$$
\begin{array}{ll}
d_{S}\left(\theta, z_{*}\right) & \\
=\frac{1}{\left(\begin{array}{c}
N \\
3
\end{array}\right)} \sum_{1 \leq n_{1}<n_{2}<n_{3} \leq N} & \left(\mathbb{1}\left\{r_{n_{1}}(\theta)>0, r_{n_{2}}(\theta)<0, r_{n_{3}}(\theta)>0\right\}\right. \\
& \left.+\mathbb{1}\left\{r_{n_{1}}(\theta)<0, r_{n_{2}}(\theta)>0, r_{n_{3}}(\theta)<0\right\}\right) .
\end{array}
$$

If $\theta$ is the true parameter, then

$$
\begin{array}{ll}
d_{S}\left(\theta, z_{*}\right) & \\
=\frac{1}{\left(\begin{array}{c}
N \\
3
\end{array}\right)} \sum_{1 \leq n_{1}<n_{2}<n_{3} \leq N} & \left(\mathbb{1}\left\{e_{n_{1}}>0, e_{n_{2}}<0, e_{n_{3}}>0\right\}\right. \\
& \left.+\mathbb{1}\left\{e_{n_{1}}<0, e_{n_{2}}>0, e_{n_{3}}<0\right\}\right),
\end{array}
$$

where $e_{n}$ is the realization of the error $E_{n}$. However, this is not a $U$-statistic anymore since $\mathbb{1}\left\{e_{n_{1}}>0, e_{n_{2}}<0, e_{n_{3}}>0\right\}+\mathbb{1}\left\{e_{n_{1}}<0, e_{n_{2}}>0, e_{n_{3}}<0\right\}$ is not a symmetric kernel. Hence, we need the asymptotic distribution for this case which is derived in the next section.

Having the asymptotic distribution of $N\left(d_{S}\left(\theta, Z_{*}\right)-\frac{1}{2}\right)$ under $\theta$ with $\alpha$-quantile $q_{\alpha}$, a simple asymptotic $\alpha$-level test for the hypothesis $H_{0}: \theta \in \Theta_{0}$, where $\Theta_{0}$ is a subset of $[0, \infty) \times(1, \infty)$, is (see Müller 2005):

$$
\text { reject } H_{0} \text { if } \sup _{\theta \in \Theta_{0}}\left(N\left(d_{S}\left(\theta, z_{*}\right)-\frac{1}{4}\right)\right) \text { is smaller than } q_{\alpha} \text {, }
$$

i.e. the depths of all parameters of the hypotheses are too small.

\section{Asymptotic Distribution of the Simplicial DePth}

Even though the statistic $d_{S}\left(\theta, z_{*}\right)$ is not an ordinary $U$-statistic due to the lack of symmetry of the kernel, its asymptotics can be obtained similarly to the derivation of of the limit distribution of first-order degenerate $U$-statistics. First, we define several functions related to the summands of the statistic $d_{S}\left(\theta, z_{*}\right)$ by

$$
\begin{gathered}
h(x, y, z)=\mathbb{1}\{x>0, y<0, z>0\}+\mathbb{1}\{x<0, y>0, z<0\}, \\
h_{1}(x)=\operatorname{E} h\left(x, E_{2}, E_{3}\right), \quad h_{2}(y)=\operatorname{E} h\left(E_{1}, y, E_{3}\right), \quad h_{3}(z)=\operatorname{E} h\left(E_{1}, E_{2}, z\right), \\
h_{1,2}(x, y)=\operatorname{E} h\left(x, y, E_{3}\right), \quad h_{1,3}(x, z)=\operatorname{Eh}\left(x, E_{2}, z\right), \\
h_{2,3}(y, z)=\operatorname{E} h\left(E_{1}, y, z\right) .
\end{gathered}
$$

Note that

$$
h_{1}(x)=\frac{1}{4}(\mathbb{1}\{x<0\}+\mathbb{1}\{x>0\})=\frac{1}{4}=h_{2}(y)=h_{3}(z) \quad \text { a.s. }
$$

which can be compared to first-order degeneracy of $U$-statistics since straight-forward calculations yield $\operatorname{var}\left(h_{i, j}\left(E_{1}, E_{2}\right)\right)=1 / 16>0$. The latter relations will be important auxiliary results for the derivation of the limit distribution of simplicial depth in the $\mathrm{AR}(1)$ setting. Moreover, we will make use of the following approximation. 
Lemma 3.1. Under the afore-mentioned assumptions,

$$
\begin{array}{ll}
N\left(d_{S}\left(\theta, Z_{*}\right)-\frac{1}{4}\right) & \\
=\frac{N}{\left(\begin{array}{c}
N \\
3
\end{array}\right)} \sum_{1 \leq n_{1}<n_{2}<n_{3} \leq N} & \left(h_{1,2}\left(E_{n_{1}}, E_{n_{2}}\right)+h_{1,3}\left(E_{n_{1}}, E_{n_{3}}\right)\right. \\
& \left.+h_{2,3}\left(E_{n_{2}}, E_{n_{3}}\right)-\frac{3}{4}\right)+o_{P}(1) .
\end{array}
$$

Proof. We have to show asymptotic negligibility of

$$
\begin{aligned}
\frac{N}{\left(\begin{array}{c}
N \\
3
\end{array}\right)} & \sum_{1 \leq n_{1}<n_{2}<n_{3} \leq N}\left(h\left(E_{n_{1}}, E_{n_{2}}, E_{n_{3}}\right)-\frac{1}{4}\right. \\
- & {\left.\left[h_{1,2}\left(E_{n_{1}}, E_{n_{2}}\right)+h_{1,3}\left(E_{n_{1}}, E_{n_{3}}\right)+h_{2,3}\left(E_{n_{2}}, E_{n_{3}}\right)-\frac{3}{4}\right]\right) . }
\end{aligned}
$$

As the expectation of this quantity is equal to zero, it remains to show that its variance tends to zero as $N \rightarrow \infty$. The latter is given by

$$
\begin{aligned}
& \frac{N^{2}}{\left(\begin{array}{c}
N \\
3
\end{array}\right)^{2}} \sum_{1 \leq n_{1}<n_{2}<n_{3} \leq N} \sum_{1 \leq \bar{n}_{1}<\bar{n}_{2}<\bar{n}_{3} \leq N} \mathrm{E}\left\{\left(h\left(E_{n_{1}}, E_{n_{2}}, E_{n_{3}}\right)+\frac{1}{2}-\left[h_{1,2}\left(E_{n_{1}}, E_{n_{2}}\right)\right.\right.\right. \\
& \left.\left.+h_{1,3}\left(E_{n_{1}}, E_{n_{3}}\right)+h_{2,3}\left(E_{n_{2}}, E_{n_{3}}\right)\right]\right) \\
& \times\left(h\left(E_{\bar{n}_{1}}, E_{\bar{n}_{2}}, E_{\bar{n}_{3}}\right)+\frac{1}{2}-\left[h_{1,2}\left(E_{\bar{n}_{1}}, E_{\bar{n}_{2}}\right)\right.\right. \\
& \left.\left.\left.+h_{1,3}\left(E_{\bar{n}_{1}}, E_{\bar{n}_{3}}\right)+h_{2,3}\left(E_{\bar{n}_{2}}, E_{\bar{n}_{3}}\right)\right]\right)\right\} .
\end{aligned}
$$

The number of summands with $n_{1}=\bar{n}_{1}, n_{2}=\bar{n}_{2}, n_{3}=\bar{n}_{3}$ is of order $O\left(N^{3}\right)$ and therefore the corresponding term asymptotically negligible as the factor in front of the sum is of order $O\left(N^{-4}\right)$. Moreover, note that due to the increasing ordering of the indices it cannot happen that four or more indices coincide. Therefore only three cases remain:

(1) All indices are different from each other.

All these summands are equal to zero by the i.i.d. assumptions on the involved random variables.

(2) Exactly two indices coincide.

Examplarily, we consider the case $n_{1}=\bar{n}_{2}$. All remaining pairs can be treated 
in a similar manner and are therefore skipped here. We obtain

$$
\begin{aligned}
& \mathrm{E}\left\{\left(h\left(E_{n_{1}}, E_{n_{2}}, E_{n_{3}}\right)+\frac{1}{2}-\left[h_{1,2}\left(E_{n_{1}}, E_{n_{2}}\right)\right.\right.\right. \\
+ & \left.\left.h_{1,3}\left(E_{n_{1}}, E_{n_{3}}\right)+h_{2,3}\left(E_{n_{2}}, E_{n_{3}}\right)\right]\right) \\
\times & \left(h\left(E_{\bar{n}_{1}}, E_{n_{1}}, E_{\bar{n}_{3}}\right)+\frac{1}{2}-\left[h_{1,2}\left(E_{\bar{n}_{1}}, E_{n_{1}}\right)\right.\right. \\
+ & \left.\left.\left.h_{1,3}\left(E_{\bar{n}_{1}}, E_{\bar{n}_{3}}\right)+h_{2,3}\left(E_{n_{1}}, E_{\bar{n}_{3}}\right)\right]\right)\right\} \\
= & \mathrm{E}\left\{\left(h\left(E_{n_{1}}, E_{n_{2}}, E_{n_{3}}\right)-\left[h_{1,2}\left(E_{n_{1}}, E_{n_{2}}\right)+h_{1,3}\left(E_{n_{1}}, E_{n_{3}}\right)\right]\right)\right. \\
& \times\left(h\left(E_{\bar{n}_{1}}, E_{n_{1}}, E_{\bar{n}_{3}}\right)+\frac{1}{2}-\left[h_{1,2}\left(E_{\bar{n}_{1}}, E_{n_{1}}\right)\right.\right. \\
+ & \left.\left.\left.0, h_{1,3}\left(E_{\bar{n}_{1}}, E_{\bar{n}_{3}}\right)+h_{2,3}\left(E_{n_{1}}, E_{\bar{n}_{3}}\right)\right]\right)\right\} \\
& \left.-h_{1}\left(E_{n_{1}}\right) h_{2}\left(E_{n_{1}}\right)-\frac{1}{8}+h_{1}\left(E_{n_{1}}\right) h_{2}\left(E_{n_{1}}\right)+\frac{1}{16}+h_{1}\left(E_{n_{1}}\right) h_{2}\left(E_{n_{1}}\right)\right\} \\
= & \mathrm{E}\left\{h_{1}\left(E_{n_{1}}\right) h_{2}\left(E_{n_{1}}\right)+\frac{1}{8}-h_{1}\left(E_{n_{1}}\right) h_{2}\left(E_{n_{1}}\right)-\frac{1}{16}-h_{1}\left(E_{n_{1}}\right) h_{2}\left(E_{n_{1}}\right)\right. \\
& -h_{1}\left(E_{n_{1}}\right) h_{2}\left(E_{n_{1}}\right)-\frac{1}{8}+h_{1}\left(E_{n_{1}}\right) h_{2}\left(E_{n_{1}}\right)+\frac{1}{16}+h_{1}\left(E_{n_{1}}\right) h_{2}\left(E_{n_{1}}\right) \\
& \\
& \\
& \\
& \\
& \\
& \\
&
\end{aligned}
$$

where the second equality is obtained by conditioning on $E_{n_{1}}$ and using the tower property of conditional expectation. The last equality follows from $h_{1}\left(E_{n_{1}}\right) \equiv h_{2}\left(E_{n_{1}}\right) \equiv 1 / 4$ a.s..

(3) Two pairs of indices coincide.

Examplarily, we consider the case $n_{1}=\bar{n}_{2}, n_{2}=\bar{n}_{3}$. All remaining combinations can again be treated in a similar manner and are therefore skipped 
here. We proceed as in the previous case and get

$$
\begin{aligned}
& \mathrm{E}\left\{\left(h\left(E_{n_{1}}, E_{n_{2}}, E_{n_{3}}\right)+\frac{1}{2}-\left[h_{1,2}\left(E_{n_{1}}, E_{n_{2}}\right)\right.\right.\right. \\
&\left.\left.+h_{1,3}\left(E_{n_{1}}, E_{n_{3}}\right)+h_{2,3}\left(E_{n_{2}}, E_{n_{3}}\right)\right]\right) \\
& \quad \times\left(h\left(E_{\bar{n}_{1}}, E_{n_{1}}, E_{n_{2}}\right)+\frac{1}{2}-\left[h_{1,2}\left(E_{\bar{n}_{1}}, E_{n_{1}}\right)\right.\right. \\
&+\left.\left.\left.h_{1,3}\left(E_{\bar{n}_{1}}, E_{n_{2}}\right)+h_{2,3}\left(E_{n_{1}}, E_{n_{2}}\right)\right]\right)\right\} \\
&= \mathrm{E}\left\{h_{1,2}\left(E_{n_{1}}, E_{n_{2}}\right) h_{2,3}\left(E_{n_{1}}, E_{n_{2}}\right)+\frac{1}{8}\right. \\
&-h_{1}\left(E_{n_{1}}\right) h_{2}\left(E_{n_{1}}\right)-h_{2}\left(E_{n_{2}}\right) h_{3}\left(E_{n_{2}}\right)-h_{1,2}\left(E_{n_{1}}, E_{n_{2}}\right) h_{2,3}\left(E_{n_{1}}, E_{n_{2}}\right) \\
&-h_{1,2}\left(E_{n_{1}}, E_{n_{2}}\right) h_{2,3}\left(E_{n_{1}}, E_{n_{2}}\right)-\frac{1}{8} \\
&+h_{1}\left(E_{n_{1}}\right) h_{2}\left(E_{n_{1}}\right)+h_{2}\left(E_{n_{2}}\right) h_{3}\left(E_{n_{2}}\right)+h_{1,2}\left(E_{n_{1}}, E_{n_{2}}\right) h_{2,3}\left(E_{n_{1}}, E_{n_{2}}\right) \\
&-h_{1}\left(E_{n_{1}}\right) h_{2}\left(E_{n_{1}}\right)-\frac{1}{8}+h_{1}\left(E_{n_{1}}\right) h_{2}\left(E_{n_{1}}\right)+\frac{1}{16}+h_{1}\left(E_{n_{1}}\right) h_{2}\left(E_{n_{1}}\right) \\
&\left.-h_{2}\left(E_{n_{2}}\right) h_{3}\left(E_{n_{2}}\right)-\frac{1}{8}+\frac{1}{16}+h_{2}\left(E_{n_{2}}\right) h_{3}\left(E_{n_{2}}\right)+h_{2}\left(E_{n_{2}}\right) h_{3}\left(E_{n_{2}}\right)\right\} \\
&= 0 .
\end{aligned}
$$

Thus, the variance of the remainder term tends to zero which completes the proof.

In order to derive the asymptotics of $N\left(d_{S}\left(\theta, Z_{*}\right)-1 / 4\right)$ it remains to investigate

$$
\begin{aligned}
& \frac{N}{\left(\begin{array}{c}
N \\
3
\end{array}\right)} \sum_{1 \leq n_{1}<n_{2}<n_{3} \leq N}\left(h_{1,2}\left(E_{n_{1}}, E_{n_{2}}\right)+h_{1,3}\left(E_{n_{1}}, E_{n_{3}}\right)+h_{2,3}\left(E_{n_{2}}, E_{n_{3}}\right)-\frac{3}{4}\right) \\
& =\frac{N}{2\left(\begin{array}{c}
N \\
3
\end{array}\right)}\left\{\sum_{1 \leq n_{1} \neq n_{2} \leq N}\left(N-\max \left\{n_{1}, n_{2}\right\}\right)\left[h_{1,2}\left(E_{n_{1}}, E_{n_{2}}\right)-\frac{1}{4}\right]\right. \\
& \quad+\sum_{1 \leq n_{1} \neq n_{3} \leq N}\left(\max \left\{n_{1}, n_{3}\right\}-\min \left\{n_{1}, n_{3}\right\}-1\right)\left[h_{1,3}\left(E_{n_{1}}, E_{n_{3}}\right)-\frac{1}{4}\right] \\
& \left.\quad+\sum_{1 \leq n_{2} \neq n_{3} \leq N}\left(\min \left\{n_{2}, n_{3}\right\}-1\right)\left[h_{2,3}\left(E_{n_{2}}, E_{n_{3}}\right)-\frac{1}{4}\right]\right\},
\end{aligned}
$$


where we used that $h_{1,2}, h_{1,3}$, and $h_{2,3}$, separately, are symmetric.

Now, invoking the fact that

$$
\sum_{1 \leq n_{1} \neq n_{2} \leq N} \min \left\{n_{1}, n_{2}\right\} h_{1,2}\left(E_{n_{1}}, E_{n_{2}}\right)=\sum_{1 \leq n_{2} \neq n_{3} \leq N} \min \left\{n_{2}, n_{3}\right\} h_{2,3}\left(E_{n_{2}}, E_{n_{3}}\right)
$$

and $N^{-2} \sum_{1 \leq n_{1} \neq n_{2} \leq N}\left(h_{1,2}\left(E_{n_{1}}, E_{n_{2}}\right)+h_{1,3}\left(E_{n_{1}}, E_{n_{2}}\right)-\frac{1}{2}\right) \longrightarrow 0$ almost surely with the SLLN, we obtain that the limit distribution of

$N\left(d_{S}\left(\theta, Z_{*}\right)-1 / 4\right)$ is asymptotically equivalent with

$$
\begin{aligned}
U_{n}=\frac{3}{N} \sum_{1 \leq n_{1} \neq n_{2} \leq N}\left[h_{1,2}\left(E_{n_{1}}, E_{n_{2}}\right)-\frac{1}{4}\right] \\
+\frac{3}{N^{2}} \sum_{1 \leq n_{1} \neq n_{2} \leq N}\left(\max \left\{n_{1}, n_{2}\right\}-\min \left\{n_{1}, n_{2}\right\}\right) \\
\cdot\left[h_{1,3}\left(E_{n_{1}}, E_{n_{2}}\right)-h_{1,2}\left(E_{n_{1}}, E_{n_{2}}\right)\right] .
\end{aligned}
$$

Now, we invoke a spectral decomposition of the remaining functions in order to separate the the variables $E_{n_{1}}$ and $E_{n_{2}}$ in a multiplicative manner. The spectral decomposition of $h_{1,2}-1 / 4$ is provided in Kustosz and Müller (2014a. Proof of Theorem 2). The corresponding eigenfunction is given by $\Phi(x)=\mathbb{1}\{x<0\}-\mathbb{1}\{x>0\}$ and the eigenvalue is $-1 / 4$. That is $h_{1,2}(x, y)-1 / 4=-\Phi(x) \Phi(y) / 4$. Similarly, we obtain $h_{1,3}(x, y)-h_{1,2}(x, y)=\Phi(x) \Phi(y) / 2$. Since $\mathrm{E}\left[\Phi^{2}\left(E_{1}\right)\right]=1$, the SLLN implies

$$
\begin{aligned}
U_{n} & =\frac{3}{2 N} \sum_{1 \leq n_{1} \neq n_{2} \leq N}\left(\frac{\left|n_{1}-n_{2}\right|}{N}-\frac{1}{2}\right) \Phi\left(E_{n_{1}}\right) \Phi\left(E_{n_{2}}\right) \\
& =\frac{3}{2 N} \sum_{n_{1}, n_{2}=1}^{N}\left(\frac{\left|n_{1}-n_{2}\right|}{N}-\frac{1}{2}\right) \Phi\left(E_{n_{1}}\right) \Phi\left(E_{n_{2}}\right)+\frac{3}{4}+o_{P}(1)
\end{aligned}
$$

Even though we separated the involved random variables in a multiplicative manner, the application of a CLT to determine the limit of the first summand is not feasible yet because of the weighting factor $\left|n_{1}-n_{2}\right| / N-1 / 2$. We solve this problem by a 
convolution-based representation of the absolute value function on the interval $[-1,1]$,

$$
\begin{aligned}
V_{n}:=\frac{3}{2 N} \sum_{n_{1}, n_{2}=1}^{N}\left(\frac{\left|n_{1}-n_{2}\right|}{N}-\frac{1}{2}\right) \Phi\left(E_{n_{1}}\right) \Phi\left(E_{n_{2}}\right) \\
=\frac{3}{2 N} \sum_{n_{1}, n_{2}=1}^{N}\left(\frac{1}{2}-\int_{-\infty}^{\infty} \mathbb{1}_{(-0.5,0.5]}(t) \mathbb{1}_{(-0.5,0.5]}\left(\frac{n_{1}-n_{2}}{N}-t\right) d t\right) \\
=\frac{3}{2 N} \sum_{n_{1}, n_{2}=1}^{N}\left(\frac{1}{2}-\int_{-\infty}^{\infty} \mathbb{1}_{(-0.5,0.5]}\left(\frac{n_{1}}{N}-t\right) \mathbb{1}_{(-0.5,0.5]}\left(\frac{n_{2}}{N}-t\right) d t\right) \\
=\frac{3}{4}\left(\frac{1}{\sqrt{N}} \sum_{n_{1}=1}^{N} \Phi\left(E_{n_{2}}\right) \Phi\left(E_{n_{2}}\right)\right. \\
-\frac{3}{2} \int_{-2}^{2}\left(\frac{1}{\sqrt{N}} \sum_{n_{1}=1}^{N} \mathbb{1}_{(-0.5,0.5]}\left(\frac{n_{1}}{N}-t\right) \Phi\left(E_{n_{1}}\right)\right)^{2} d t
\end{aligned}
$$

To sum up

$$
N\left(d_{S}\left(\theta, Z_{*}\right)-\frac{1}{4}\right)=V_{n}+\frac{3}{4}+o_{P}(1)
$$

and the limit distribution of $V_{n}$ can be deduced by the continuous mapping theorem if the bivariate process

$$
\begin{aligned}
X_{N} & =\left(X_{N, 1}, X_{N, 2}\right)^{\top} \\
& =\left(\left(\frac{1}{\sqrt{N}} \sum_{n_{1}=1}^{N} \mathbb{1}_{(-0.5,0.5]}\left(\frac{n_{1}}{N}-t\right) \Phi\left(E_{n_{1}}\right), \frac{1}{\sqrt{N}} \sum_{n_{1}=1}^{N} \Phi\left(E_{n_{1}}\right)\right)^{\top}\right)_{t \in[-2,2]}
\end{aligned}
$$

converges in distribution to some continuous limiting process with respect to the uniform norm.

Lemma 3.2. Under the assumptions above

$$
X_{N} \stackrel{d}{\longrightarrow} X
$$

where $X=\left(X_{1}, X_{2}\right)^{\top}$ is a centered Gaussian process on $[-2,2]$ with continuous paths and the covariance structure

$$
\begin{aligned}
& \operatorname{Cov}(X(s), X(t)) \\
& =\left(\begin{array}{cc}
\int_{0}^{1} \mathbb{1}_{(-0.5,0.5]}(x-s) \mathbb{1}_{(-0.5,0.5]}(x-t) d x & \int_{0}^{1} \mathbb{1}_{(-0.5,0.5]}(x-s) d x \\
\int_{0}^{1} \mathbb{1}_{(-0.5,0.5]}(x-t) d x & 1
\end{array}\right) .
\end{aligned}
$$

In Figure 1, a simulation of a path of this bivariate process is depicted. The solid line represents the variable $X_{1}(t)$ which starts in 0 at $t=-0.5$ and returns to 0 at $t=1.5$. The dashed line is a simulation of $X_{2}(t)$. This process is a draw from a 
$N(0,1)$ distribution and is constant over time. Note, that the two processes meet at $t=0.5$, due to the underlying covariance structure.

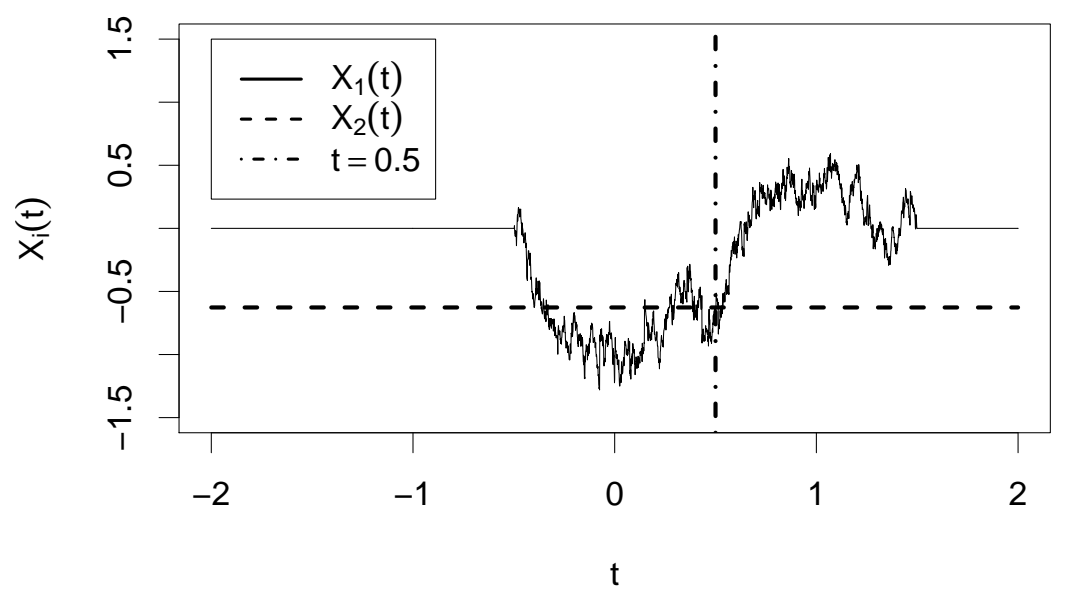

FiguRE 1. Simulation of a path of the limit process

Proof. To prove Lemma 3.2 we apply Theorem 1.5.4 and Problem 1.5.3 in van der Vaart and Wellner (2000) and proceed in several steps.

(1) Convergence of the finite dimensional distributions.

We apply the multivariate Lindeberg-Feller CLT to determine the asymptotics of $\left(X_{N, 1}\left(s_{1}\right), \ldots, X_{N, 1}\left(s_{k}\right), X_{N, 2}\left(t_{1}\right), \ldots, X_{N, 2}\left(t_{l}\right)\right)$ for arbitrary $k, l \in \mathbb{N}$ and $s_{1}, \ldots, s_{k}, t_{1}, \ldots, t_{l} \in[-2,2]$. Obviously, these variables are centered, have finite variance and satisfy the Lindeberg condition. Therefore, it remains to show convergence of the entries of the covariance matrices. For $i=1, \ldots, k$ and $j=1, \ldots, l$, we get

$$
\begin{aligned}
& \frac{1}{N} \sum_{n_{1}=1}^{N} \operatorname{var}\left(\Phi\left(E_{n_{1}}\right)\right)=1 \\
& \frac{1}{N} \sum_{n_{1}=1}^{N} \operatorname{cov}\left(\mathbb{1}_{(-0.5,0.5]}\left(\frac{n_{1}}{N}-t_{j}\right) \Phi\left(E_{n_{1}}\right), \Phi\left(E_{n_{1}}\right)\right) \\
& \underset{N \rightarrow \infty}{\longrightarrow} \int_{0}^{1} \mathbb{1}_{(-0.5,0.5]}\left(x-t_{j}\right) d x \\
& \frac{1}{N} \sum_{n_{1}=1}^{N} \operatorname{cov}\left(\mathbb{1}_{(-0.5,0.5]}\left(\frac{n_{1}}{N}-s_{i}\right) \Phi\left(E_{n_{1}}\right), \mathbb{1}_{(-0.5,0.5]}\left(\frac{n_{1}}{N}-t_{j}\right) \Phi\left(E_{n_{1}}\right)\right) \\
& \underset{N \rightarrow \infty}{\longrightarrow} \int_{0}^{1} \mathbb{1}_{(-0.5,0.5]}\left(x-s_{i}\right) \mathbb{1}_{(-0.5,0.5]}\left(x-t_{j}\right) d x,
\end{aligned}
$$


in view of $\mathrm{E} \Phi\left(E_{1}\right)=0$ and $\mathrm{E}^{2}\left(E_{1}\right)=1$.

(2) A useful moment bound.

For $-2 \leq s \leq t \leq 2$, we obtain using $\mathrm{E} \Phi\left(E_{n_{1}}\right)=0, \mathrm{E}^{2}\left(E_{n_{1}}\right)=1=\mathrm{E}^{4}\left(E_{n_{1}}\right)$

$$
\begin{aligned}
& \mathrm{E}\left\|X_{N}(s)-X_{N}(t)\right\|_{1}^{4} \\
& =\mathrm{E}\left(\frac{1}{\sqrt{N}} \sum_{n_{1}=1}^{N} \Phi\left(E_{n_{1}}\right)\left[\mathbb{1}_{(-0.5,0.5]}\left(\frac{n_{1}}{N}-s\right)-\mathbb{1}_{(-0.5,0.5]}\left(\frac{n_{1}}{N}-t\right)\right]\right)^{4} \\
& =\frac{1}{N^{2}}\left(\sum_{n_{1}=1}^{N}\left|\mathbb{1}_{(-0.5,0.5]}\left(\frac{n_{1}}{N}-s\right)-\mathbb{1}_{(-0.5,0.5]}\left(\frac{n_{1}}{N}-t\right)\right|\right)^{2} \\
& +\frac{1}{N^{2}} \sum_{n_{1}=1}^{N}\left[\mathbb{1}_{(-0.5,0.5]}\left(\frac{n_{1}}{N}-s\right)-\mathbb{1}_{(-0.5,0.5]}\left(\frac{n_{1}}{N}-t\right)\right]^{4} .
\end{aligned}
$$

Noting that

$$
\left|\mathbb{1}_{(-0.5,0.5]}\left(\frac{n_{1}}{N}-s\right)-\mathbb{1}_{(-0.5,0.5]}\left(\frac{n_{1}}{N}-t\right)\right|=1
$$

for at most $2|t-s| N$ indices $n_{1}$, we end up with

$$
\mathrm{E}\left\|X_{N}(s)-X_{N}(t)\right\|_{1}^{4} \leq 4\left((t-s)^{2}+N^{-1}|t-s|\right)
$$

(3) Existence and continuity of the limiting process.

By step 1 and Kolmogorov's existence theorem, there exists a process $X$ with the above-mentioned finite-dimensional distributions. Moreover, from (3.4) we obtain by Fatou's Lemma

$$
\mathrm{E}\|X(s)-X(t)\|_{1}^{4} \leq 4(t-s)^{2}
$$

Thus, the theorem of Kolmogorov and Chentsov implies that there exists a continuous modification of $X$ that we also refer to as $X$ in the sequel.

(4) Tightness.

By van der Vaart and Wellner (2000, Theorem 1.5.6) it remains to show that for any $\epsilon, \eta>0$ there is a partition $-2=t_{0}<t_{1}<\cdots<t_{K}=2$ such that

$$
\limsup _{N \rightarrow \infty} P\left(\sup _{k=1, \ldots, K} \sup _{s, t \in\left[t_{k-1}, t_{k}\right]}\left|X_{N, 1}(s)-X_{N, 1}(t)\right|>\epsilon\right) \leq \eta
$$

since tightness of $\left(X_{N, 2}\right)_{N}$ is trivial. 
For $X_{N, 1}$ we obtain

$$
\begin{aligned}
& P\left(\sup _{k=1, \ldots, K} \sup _{s, t \in\left[t_{k-1}, t_{k}\right]}\left|X_{N, 1}(s)-X_{N, 1}(t)\right|>\epsilon\right) \\
& \leq 2 \sum_{k=1}^{K} P\left(\sup _{t \in\left[t_{k-1}, t_{k}\right]}\left|X_{N, 1}\left(t_{k-1}\right)-X_{N, 1}(t)\right|>\frac{\epsilon}{2}\right) \\
& =2 \sum_{k=1}^{K} P\left(\sup _{t \in\left[t_{k-1}, t_{k}\right]} \mid \frac{1}{\sqrt{N}} \sum_{n_{1}=1}^{N} \phi\left(E_{n_{1}}\right)\left\{\mathbb{1}_{\left(-0.5+t_{k-1},-0.5+t\right]}\left(\frac{n_{1}}{N}\right)\right.\right. \\
& \left.\left.\quad-\mathbb{1}_{\left(0.5+t_{k-1}, 0.5+t\right]}\left(\frac{n_{1}}{N}\right)\right\} \mid>\frac{\epsilon}{2}\right)
\end{aligned}
$$

For symmetry reasons we only consider

$$
\frac{1}{\sqrt{N}} \sum_{n_{1}=1}^{N} \phi\left(E_{n_{1}}\right) \mathbb{1}_{\left(-0.5+t_{k-1},-0.5+t\right]}\left(\frac{n_{1}}{N}\right)=Q_{N}(t)-Q_{N}\left(t_{k-1}\right)
$$

with $Q_{N}(t):=\frac{1}{\sqrt{N}} \sum_{n_{1}=1}^{N} \phi\left(E_{n_{1}}\right) \mathbb{1}_{(-2,-0.5+t]}\left(\frac{n_{1}}{N}\right)$. The process $Q_{N}$ has càdlàg paths and independent increments. Moreover, note that it follows from the proof of (2) and Markov's inequality that for some $\alpha, \beta>0, P\left(\mid Q_{N}(t)-\right.$ $\left.Q_{N}(s) \mid \leq \delta\right) \geq \beta$ whenever $|t-s| \leq \alpha$ and $N \geq N_{0}$. Therefore we can proceed as in the proof of Theorem V.19 in Pollard (1984) to obtain

$$
\limsup _{N \rightarrow \infty} \sum_{k=1}^{K} P\left(\sup _{t \in\left[t_{k-1}, t_{k}\right]}\left|Q_{N}(t)-Q_{N}\left(t_{k-1}\right)\right|>\frac{\epsilon}{4}\right)<\frac{\eta}{4}
$$

for a sufficiently fine equidistant grid.

To sum up, we get the following theorem on the asymptotics of simplicial depth by the continuous mapping theorem.

Theorem 3.1. Under the assumptions above

$$
N\left(d_{S}\left(\theta, Z_{*}\right)-\frac{1}{4}\right) \stackrel{d}{\longrightarrow} \frac{3}{4}+\frac{3}{4} X_{2}^{2}(0)-\frac{3}{2} \int_{-2}^{2} X_{1}^{2}(t) d t .
$$

Note, that the asymptotic distribution of the simplicial depth is not restricted to the $\mathrm{AR}(1)$ model considered in this paper. It holds for all cases where depth of a two dimensional parameter at three data points is given by alternating signs of the three residuals. This holds in several other models as shown in Kustosz and Müller (2014b). 


\section{Consistency of the tests}

Here we show consistency of the test given by 2.1 for hypotheses $H_{0}: \theta=\theta^{0}$ and $H_{0}: \theta_{1} \geq \theta_{1}^{0}$ at all relevant alternatives $\theta^{*}=\left(\theta_{0}^{*}, \theta_{1}^{*}\right)^{\top}$ by using a large upper bound of the test statistic. Thereby, a test is called consistent at $\theta^{*}$ if the power of the test at $\theta^{*}$ is converging to one for growing sample size. Hence we have to proof here

$$
\lim _{N \rightarrow \infty} P_{\theta^{*}}\left(\sup _{\theta \in \Theta_{0}}\left(N\left(d_{S}\left(\theta, Z_{*}\right)-\frac{1}{4}\right)\right)<q_{\alpha}\right)=1
$$

where $q_{\alpha}$ is the $\alpha$-quantile of the asymptotic distribution of $N\left(d_{S}\left(\theta, Z_{*}\right)-\frac{1}{4}\right)$ given by Theorem 3.1 .

Lemma 4.1. If there exists $N_{0} \in \mathbb{N}, \delta \in\left(0, \frac{1}{4}\right)$, and a bounded function $H: \mathbb{R}^{3} \rightarrow \mathbb{R}$ with

$$
\begin{aligned}
& H\left(r_{n_{1}}\left(\theta^{*}\right), r_{n_{2}}\left(\theta^{*}\right), r_{n_{3}}\left(\theta^{*}\right)\right) \\
& \geq \sup _{\theta \in \Theta_{0}}\left(\mathbb{1}\left\{r_{n_{1}}(\theta)>0, r_{n_{2}}(\theta)<0, r_{n_{3}}(\theta)>0\right\}\right. \\
& \left.\quad+\mathbb{1}\left\{r_{n_{1}}(\theta)<0, r_{n_{2}}(\theta)>0, r_{n_{3}}(\theta)<0\right\}\right)
\end{aligned}
$$

for all $n_{1}, n_{2}, n_{3}>N_{0}$ and

$$
E_{\theta^{*}}\left(H\left(r_{n_{1}}\left(\theta^{*}\right), r_{n_{2}}\left(\theta^{*}\right), r_{n_{3}}\left(\theta^{*}\right)\right)\right)<\frac{1}{4}-\delta,
$$

then the test given by (2.1) is consistent at $\theta^{*}$.

Proof. Set $M_{0}=\left\{\left(n_{1}, n_{2}, n_{3}\right) ; n_{3}>n_{2}>n_{1}>N_{0}\right\}$, then

$$
\begin{aligned}
& \sup _{\theta \in \Theta_{0}}\left(N\left(d_{S}\left(\theta, Z_{*}\right)-\frac{1}{4}\right)\right) \\
\leq & \frac{N}{\left(\begin{array}{c}
N \\
3
\end{array}\right)}\left(\sum _ { 1 \leq n _ { 1 } < n _ { 2 } < n _ { 3 } \leq N } \operatorname { s u p } _ { \theta \in \Theta _ { 0 } } \left(\mathbb{1}\left\{r_{n_{1}}(\theta)>0, r_{n_{2}}(\theta)<0, r_{n_{3}}(\theta)>0\right\}\right.\right. \\
& \left.\left.+\mathbb{1}\left\{r_{n_{1}}(\theta)<0, r_{n_{2}}(\theta)>0, r_{n_{3}}(\theta)<0\right\}\right)-\frac{1}{4}\right) \\
\leq & \frac{N}{\left(\begin{array}{c}
N \\
3
\end{array}\right)}\left(\left[\left(\begin{array}{c}
N \\
3
\end{array}\right)-\left(\begin{array}{c}
N-N_{0} \\
3
\end{array}\right)\right]\right. \\
+ & \left.\sum_{\left(n_{1}, n_{2}, n_{3}\right) \in M_{0}}\left(H\left(r_{n_{1}}\left(\theta^{*}\right), r_{n_{2}}\left(\theta^{*}\right), r_{n_{3}}\left(\theta^{*}\right)\right)-\frac{1}{4}\right)\right)=: T .
\end{aligned}
$$

Hence, we will work with $T$. To apply Chebyshev's inequality, we need upper bounds for the expectation and the variance of $T$. Since (4.2) holds on $M_{0}$ and the indicators 
are bounded on the remaining indices, we get

$$
\mathrm{E}_{\theta^{*}}(T) \leq \frac{N}{\left(\begin{array}{c}
N \\
3
\end{array}\right)}\left(\left[\left(\begin{array}{c}
N \\
3
\end{array}\right)-\left(\begin{array}{c}
N-N_{0} \\
3
\end{array}\right)+\left(\begin{array}{c}
N-N_{0} \\
3
\end{array}\right)(-\delta)\right]\right)
$$

Then there exists $N_{1}>N_{0}$ such that

$$
\frac{1}{\left(\begin{array}{c}
N \\
3
\end{array}\right)}\left(\begin{array}{c}
N-N_{0} \\
3
\end{array}\right) \geq 1-\delta \text { and } \frac{1}{\left(\begin{array}{c}
N \\
3
\end{array}\right)}\left[\left(\begin{array}{c}
N \\
3
\end{array}\right)-\left(\begin{array}{c}
N-N_{0} \\
3
\end{array}\right)\right]<\frac{\delta}{2}
$$

for all $N \geq N_{1}$ implying

$$
E_{\theta^{*}}(T) \leq N\left(\frac{\delta}{2}+(1-\delta)(-\delta)\right)=-N \delta_{0}
$$

with $\delta_{0}=\frac{1}{2} \delta-\delta^{2}>0$ since $\delta<\frac{1}{4}$. Setting

$H_{n_{1}, n_{2}, n_{3}}=H\left(r_{n_{1}}\left(\theta^{*}\right), r_{n_{2}}\left(\theta^{*}\right), r_{n_{3}}\left(\theta^{*}\right)\right)-E_{\theta^{*}}\left[H\left(r_{n_{1}}\left(\theta^{*}\right), r_{n_{2}}\left(\theta^{*}\right), r_{n_{3}}\left(\theta^{*}\right)\right)\right]$, we obtain for the variance

$$
\begin{aligned}
& \operatorname{var}_{\theta^{*}}(T)=\frac{N^{2}}{\left(\begin{array}{c}
N \\
3
\end{array}\right)^{2}} \mathrm{E}_{\theta^{*}}\left\{\left(\sum_{\left(n_{1}, n_{2}, n_{3}\right) \in M_{0}} H_{n_{1}, n_{2}, n_{3}}\right)^{2}\right\} \\
& =\frac{N^{2}}{\left(\begin{array}{c}
N \\
3
\end{array}\right)^{2}} \sum_{\left(n_{1}, n_{2}, n_{3}\right) \in M_{0}} \sum_{\left(\bar{n}_{1}, \bar{n}_{2}, \bar{n}_{3}\right) \in M_{0}} \mathrm{E}_{\theta^{*}}\left(H_{n_{1}, n_{2}, n_{3}} H_{\bar{n}_{1}, \bar{n}_{2}, \bar{n}_{3}}\right) .
\end{aligned}
$$

For $\left(\begin{array}{c}N-N_{0} \\ 3\end{array}\right)\left(\begin{array}{c}N-N_{0}-3 \\ 3\end{array}\right)$ combinations, all $n_{1}, n_{2}, n_{3}$ are different from $\bar{n}_{1}, \bar{n}_{2}, \bar{n}_{3}$, so that the independence of the residuals $r_{n}\left(\theta^{*}\right)$ implies

$\mathrm{E}_{\theta^{*}}\left(H_{n_{1}, n_{2}, n_{3}} H_{\bar{n}_{1}, \bar{n}_{2}, \bar{n}_{3}}\right)=0$ for these cases. In all other cases,

$\mathrm{E}_{\theta^{*}}\left(H_{n_{1}, n_{2}, n_{3}} H_{\bar{n}_{1}, \bar{n}_{2}, \bar{n}_{3}}\right)$ is bounded by some $b^{2}$ so that

$$
\operatorname{var}_{\theta^{*}}(T) \leq \frac{N^{2}}{\left(\begin{array}{c}
N \\
3
\end{array}\right)^{2}}\left(\left(\begin{array}{c}
N-N_{0} \\
3
\end{array}\right)^{2}-\left(\begin{array}{c}
N-N_{0} \\
3
\end{array}\right)\left(\begin{array}{c}
N-N_{0}-3 \\
3
\end{array}\right)\right) b^{2}
$$

Hence for all $\epsilon>0$, there exists $N_{2} \geq N_{1}>N_{0}$ such that $\delta_{0}+\frac{q_{\alpha}}{N_{2}}>0$ and

$$
\operatorname{var}_{\theta^{*}}(T) \leq \epsilon N^{2}\left(\delta_{0}+\frac{q_{\alpha}}{N_{2}}\right)^{2}
$$


for all $N \geq N_{2}$. Finally, Chebyshev's inequality provides for all $N \geq N_{2}$ using $\frac{q_{\alpha}}{N} \geq \frac{q_{\alpha}}{N_{2}}$

$$
\begin{aligned}
& P_{\theta^{*}}\left(\sup _{\theta \in \Theta_{0}}\left(N\left(d_{S}\left(\theta, Z_{*}\right)-\frac{1}{4}\right)\right) \geq q_{\alpha}\right) \\
& \leq P_{\theta^{*}}\left(T \geq q_{\alpha}\right) \leq P_{\theta^{*}}\left(\left|T-E_{\theta^{*}}(T)\right| \geq q_{\alpha}-E_{\theta^{*}}(T)\right) \\
& \leq P_{\theta^{*}}\left(\left|T-E_{\theta^{*}}(T)\right| \geq q_{\alpha}+N \delta_{0}\right) \leq \frac{\epsilon N^{2}\left(\delta_{0}+\frac{q_{\alpha}}{N_{2}}\right)^{2}}{N^{2}\left(\delta_{0}+\frac{q_{\alpha}}{N}\right)^{2}} \\
& \leq \frac{\epsilon\left(\delta_{0}+\frac{q_{\alpha}}{N_{2}}\right)^{2}}{\left(\delta_{0}+\frac{q_{\alpha}}{N_{2}}\right)^{2}}=\epsilon,
\end{aligned}
$$

since $q_{\alpha}<0$ (see Section 5).

The following Lemma is easy to see by induction.

Lemma 4.2. If $Y_{0}=y_{0}$ and the errors satisfy $E_{n} \geq y_{0}-\theta_{0}-\theta_{1} y_{0}+c$ for all $n$ for some $y_{0}>0$ and $c>0$, then $Y_{n}$ is strictly increasing with

$$
Y_{n} \geq\left(\sum_{k=0}^{n-1} \theta_{1}^{k}\right) c+y_{0}
$$

If, for example, the errors have a shifted Fréchet distribution as used in the simulations in Section 5, then $E_{n} \geq y_{0}-\tau y_{0}+c$ and $\operatorname{med}\left(E_{n}\right)=0$ is satisfied for some $y_{0}>0, c>0$, and $\tau>1$.

Theorem 4.1. If the errors satisfy $E_{n} \geq y_{0}-\tau y_{0}+c$ for all $n$ for some $y_{0}>0$, $c>0, \tau>1, Y_{0}=y_{0}, \Theta=[0, \infty) \times[\tau, \infty), \theta_{0}^{0} \geq 0, \theta_{1}^{0}>\tau$ and

$$
\Theta_{0}=\left\{\left(\theta_{0}, \theta_{1}\right)^{\top} \in \Theta ; \theta_{1} \geq \theta_{1}^{0}\right\} \text { or } \Theta_{0}=\left\{\left(\theta_{0}^{0}, \theta_{1}^{0}\right)^{\top}\right\}
$$

then the test given by 2.1 is consistent at all $\theta^{*} \in \Theta \backslash \Theta_{0}$.

Proof. Set $\Theta_{0}^{h}=\left\{\left(\theta_{0}, \theta_{1}\right)^{\top} \in \Theta ; \theta_{1} \geq \theta_{1}^{0}\right\}$ for the half-sided null hypotheses and $\Theta_{0}^{p}=\left\{\left(\theta_{0}^{0}, \theta_{1}^{0}\right)^{\top}\right\}$ for the point null hypothesis and use $h$ defined in 3.1 . 
If $\theta^{*} \in \Theta \backslash \Theta_{0}^{h}$ then

$$
\begin{aligned}
& \sup _{\theta \in \Theta_{0}^{h}} h\left(r_{n_{1}}(\theta), r_{n_{2}}(\theta), r_{n_{3}}(\theta)\right) \\
& \leq \sup _{\theta \in \Theta_{0}^{h}}\left(\mathbb{1}\left\{r_{n_{1}}(\theta)>0\right\}+\mathbb{1}\left\{r_{n_{2}}(\theta)>0\right\}\right) \\
& =\sup _{\theta \in \Theta_{0}^{h}}\left(\mathbb{1}\left\{r_{n_{1}}\left(\theta^{*}\right)>\theta_{0}-\theta_{0}^{*}+\left(\theta_{1}-\theta_{1}^{*}\right) Y_{n_{1}-1}\right\}\right. \\
& \left.\quad+\mathbb{1}\left\{r_{n_{2}}\left(\theta^{*}\right)>\theta_{0}-\theta_{0}^{*}+\left(\theta_{1}-\theta_{1}^{*}\right) Y_{n_{2}-1}\right\}\right) \\
& \leq \quad \mathbb{1}\left\{r_{n_{1}}\left(\theta^{*}\right)>-\theta_{0}^{*}+\left(\theta_{1}^{0}-\theta_{1}^{*}\right) Y_{n_{1}-1}\right\} \\
& \quad+\mathbb{1}\left\{r_{n_{2}}\left(\theta^{*}\right)>-\theta_{0}^{*}+\left(\theta_{1}^{0}-\theta_{1}^{*}\right) Y_{n_{2}-1}\right\}
\end{aligned}
$$

since $\theta_{0} \geq 0$ and $\theta_{1} \geq \theta_{1}^{0}$ for $\theta=\left(\theta_{0}, \theta_{1}\right)^{\top} \in \Theta_{0}^{h}$. According to Lemma 4.2 , for all $\gamma>0$ there exist $N_{0}$ such that

$$
Y_{n} \geq\left(\sum_{k=0}^{n-1} \theta_{1}^{k}\right) c+y_{0} \geq \gamma
$$

for all $N \geq N_{0}$. In particular $\gamma$ can be chosen such that $-\theta_{0}^{*}+\left(\theta_{1}^{0}-\theta_{1}^{*}\right) \gamma>k$ and $P_{\theta^{*}}\left(r_{n}\left(\theta^{*}\right)>k\right)<\frac{1}{2}\left(\frac{1}{4}-\delta\right)$ with $\delta \in\left(0, \frac{1}{4}\right)$ since $\theta_{1}^{0}>\theta_{1}^{*}$. Setting

$$
H\left(r_{n_{1}}\left(\theta^{*}\right), r_{n_{2}}\left(\theta^{*}\right), r_{n_{3}}\left(\theta^{*}\right)\right)=\mathbb{1}\left\{r_{n_{1}}\left(\theta^{*}\right)>k\right\}+\mathbb{1}\left\{r_{n_{2}}\left(\theta^{*}\right)>k\right\},
$$

Conditions (4.1) and 4.2) are satisfied, so that consistency holds for all $\theta^{*} \in \Theta \backslash \Theta_{0}^{h}$.

If $\theta^{*} \in \Theta \backslash \Theta_{0}^{p}$ and $\theta_{1}^{0}>\theta_{1}^{*}$, then consistency at $\theta^{*}$ follows as above. If $\theta_{1}^{0}<\theta_{1}^{*}$, then there exists $\gamma>0$ with $\theta_{0}^{0}-\theta_{0}^{*}+\left(\theta_{1}^{0}-\theta_{1}^{*}\right) \gamma<-k$ and $P_{\theta^{*}}\left(r_{n}\left(\theta^{*}\right)<-k\right)<\frac{1}{2}\left(\frac{1}{4}-\delta\right)$ so that

$$
H\left(r_{n_{1}}\left(\theta^{*}\right), r_{n_{2}}\left(\theta^{*}\right), r_{n_{3}}\left(\theta^{*}\right)\right)=\mathbb{1}\left\{r_{n_{1}}\left(\theta^{*}\right)<-k\right\}+\mathbb{1}\left\{r_{n_{2}}\left(\theta^{*}\right)<-k\right\}
$$

satisfies Conditions (4.1) and (4.2). If $\theta_{1}^{0}=\theta_{1}^{*}$, then $k:=\theta_{0}^{0}-\theta_{0}^{*} \neq 0$ and

$$
\begin{aligned}
& h\left(r_{n_{1}}(\theta), r_{n_{2}}(\theta), r_{n_{3}}(\theta)\right) \\
& =\quad \mathbb{1}\left\{r_{n_{1}}\left(\theta^{*}\right)>k, r_{n_{2}}\left(\theta^{*}\right)<k, r_{n_{3}}\left(\theta^{*}\right)>k\right\} \\
& \quad \quad+\mathbb{1}\left\{r_{n_{1}}\left(\theta^{*}\right)<k, r_{n_{2}}\left(\theta^{*}\right)>k, r_{n_{3}}\left(\theta^{*}\right)<k\right\} \\
& =: \quad H\left(r_{n_{1}}\left(\theta^{*}\right), r_{n_{2}}\left(\theta^{*}\right), r_{n_{3}}\left(\theta^{*}\right)\right) .
\end{aligned}
$$

Since $p(1-p) p+(1-p) p(1-p)=p(1-p)<\frac{1}{4}$ for all $p \neq \frac{1}{2}$ and $p=P_{\theta^{*}}\left(r_{n}\left(\theta^{*}\right)<k\right) \neq \frac{1}{2}$, Condition 4.2 is also satisfied. Hence consistency holds for all $\theta^{*} \in \Theta \backslash \Theta_{0}^{p}$ as well. 


\section{Simulation of the POWER OF the test}

Since confidence sets can be constructed from point hypotheses, we consider here only the most important hypothesis $H_{0}: \theta=\theta^{0}$. To simulate the power of the test based on the simplicial depth for this hypothesis, approximate quantiles of the asymptotic distribution given by Theorem 3.1 were determined, and an efficient algorithm for calculating the test statistic was developed.

\subsection{Quantiles of the asymptotic distribution.}

To calculate approximate quantiles, we use an equidistant partition of $[-2,2]$ defined by $t_{0}=-2, t_{i+1}=t_{i}+h$ and $t_{K}=2$ with $h=0.001$ and generate 200000 repetitions of the process $x\left(t_{0}\right), x\left(t_{1}\right), \ldots, x\left(t_{K}\right)$. According to Lemma $3.2,\left(X\left(t_{0}\right)^{\top}, X\left(t_{1}\right)^{\top}, \ldots\right.$, $\left.X\left(t_{K}\right)^{\top}\right)^{\top}$ has a multivariate normal distribution with a degenerated $2 K \times 2 K$ covariance matrix. To avoid degeneracy in some cases and to reduce the computational costs, we simulate $\left(x_{1}\left(t_{1}\right), x_{1}\left(t_{2}\right), \ldots, x_{1}\left(t_{K}\right)\right)^{\top}$ from the conditional distribution of $\left(X_{1}\left(t_{1}\right), X_{1}\left(t_{2}\right), \ldots, X_{1}\left(t_{K}\right)\right)^{\top}$ given $\left(X_{2}\left(t_{1}\right), X_{2}\left(t_{2}\right), \ldots, X_{2}\left(t_{K}\right)\right)^{\top}=x$

where $x=\left(x_{2}\left(t_{0}\right), \ldots, x_{2}\left(t_{0}\right)\right)^{\top}$ results from one realization of the standard normal distribution. Then an approximation of the integrated limit process is given by

$$
W_{K}=\frac{3}{4}+\frac{3}{4} x_{2}\left(t_{0}\right)^{2}-\frac{3}{2} \sum_{k=0}^{K-1} \frac{1}{2}\left(x_{1}\left(t_{k+1}\right)^{2}+x_{1}\left(t_{k}\right)^{2}\right)\left(t_{k+1}-t_{k}\right) .
$$

Taking the empirical quantiles of $W_{K}$, yields the approximate quantiles of the asymptotic distribution presented in Table 1. A complete quantile plot is depicted in Figure 2.

\begin{tabular}{l|c|c|c|c|c|c|}
$\alpha$ & 0.005 & 0.01 & 0.025 & 0.05 & 0.1 & 0.2 \\
\hline$q_{\alpha}$ & -2.6543 & -2.2404 & -1.6792 & -1.2545 & -0.8271 & -0.4032 \\
TABLE 1. Quantiles of the integrated Gaussian process
\end{tabular}

\subsection{Calculation of the test statistic.}

To evaluate the full depth statistic $d_{S}\left(\theta, z_{*}\right)$, we apply parallel computing and matrix based operations which reduce the computational costs to order $N^{2}$ compared to the naive calculation with order $N^{3}$. Therefore we use tilted triangular matrices and Hankel matrices to calculate depth with one residual fixed in an efficient way. In particular the new algorithm allows a parallel computation of the remaining loop. All computations are performed in $R$ (see, R Core Team, 2013) with the packages for multi-core computation (see Yu 2002, Tierney 2013, Venables and Ripley 2002) and a package for fast matrix calculations (see Novometsky 2012).

5.3. Simulation study. We compare the test based on simplicial depth for $H_{0}$ : $\theta=\theta^{0}$ using $\theta^{0}=\left(\theta_{0}^{0}, \theta_{1}^{0}\right)^{\top}=(0.2,1.01)^{\top}$ with five other tests. Three of them are simplified depth statistics using partial evaluation of the full simplicial depth and were proposed in Kustosz and Müller (2014b). This simplifies the calculation and 


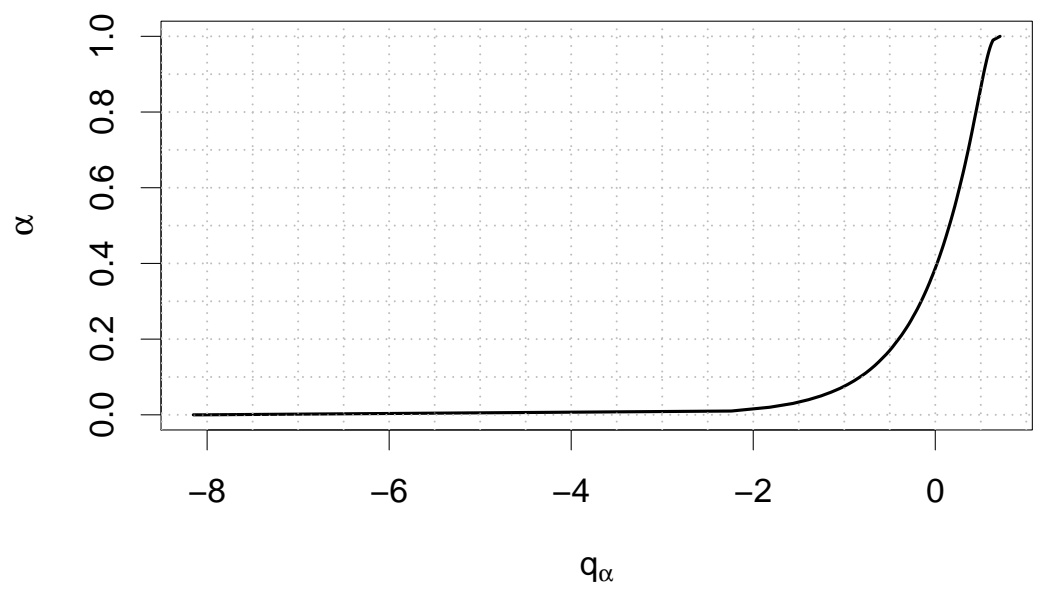

Figure 2. Quantiles of the approximate distribution of $N\left(d_{S}\left(\theta, Z_{*}\right)-\frac{1}{4}\right)$

allows a simple derivation of limit distributions which is the normal distribution. The simplified statistics are defined by

$$
\begin{gathered}
d_{S}^{1}\left(\theta, z_{*}\right)=\frac{1}{\left\lfloor\frac{N}{3}\right\rfloor} \sum_{n=1}^{\left\lfloor\frac{N}{3}\right\rfloor} \mathbb{1}\left\{r_{3 n-2}(\theta)>0, r_{3 n-1}(\theta)<0, r_{3 n}(\theta)>0\right\} \\
+\mathbb{1}\left\{r_{3 n-2}(\theta)<0, r_{3 n-1}(\theta)>0, r_{3 n}(\theta)<0\right\}, \\
d_{S}^{2}\left(\theta, z_{*}\right)=\frac{1}{\left\lfloor\frac{N-1}{2}\right\rfloor} \sum_{n=1}^{\left\lfloor\frac{N-1}{2}\right\rfloor} \mathbb{1}\left\{r_{n}(\theta)>0, r_{\left\lfloor\frac{N+1}{2}\right\rfloor}(\theta)<0, r_{N-n+1}(\theta)>0\right\} \\
+\mathbb{1}\left\{r_{n}(\theta)<0, r_{\left\lfloor\frac{N+1}{2}\right\rfloor}(\theta)>0, r_{N-n+1}(\theta)>0\right\}, \\
d_{S}^{3}\left(\theta, z_{*}\right)=\frac{1}{N-2} \sum_{n=1}^{N-2} \mathbb{1}\left\{r_{n}(\theta)>0, r_{n+1}(\theta)<0, r_{n+2}(\theta)>0\right\} \\
+\mathbb{1}\left\{r_{n}(\theta)<0, r_{n+1}(\theta)>0, r_{n+2}(\theta)<0\right\} .
\end{gathered}
$$

The other two tests are the simple sign test defined by

$$
T_{s}\left(\theta, z_{*}\right)=\frac{1}{\sqrt{N}} \sum_{n=1}^{N} \operatorname{sign}\left(r_{n}(\theta)\right)
$$

which is described in Huggins (1989) for stochastic processes, and an OLS test based on the limit distributions derived in Wang and Yu (2013). The sign test uses the exact error distribution of the residual signs when the $\operatorname{med}\left(E_{n}\right)=0$ assumption holds. For the OLS test we define critical values based on the asymptotic independence of the marginal estimators. Since we do not know the exact error distribution, we apply the OLS test assuming normal errors in all examples. We evaluate the power of the six 
tests on a grid defined by $\theta_{0} \in[-0.15,0.52]$ with mesh size 0.01 and $\theta_{1} \in[1,1.021]$ with mesh size 0.0003 . For each grid point, we simulate $R=100$ processes of length $N=100$ with the underlying parameter combination and with starting value $y_{0}=15$.

We used three different distributions for the errors: a normal distribution with mean zero and variance 0.01, a contaminated normal distribution given by $A_{n}+P_{n}$. $B_{n}$, whereby $A_{n} \sim \mathcal{N}(0,0.1), B_{n} \sim \mathcal{N}(5,1)$ and $P_{n} \sim \operatorname{Pois}(5 / 100)$ are independent random variables for each $n$, and a Fréchet distribution defined by the density

$$
f_{\alpha, \beta, \gamma}(x)=\frac{\gamma}{\alpha}\left(\frac{x-\beta}{\alpha}\right)^{-1-\gamma} \exp \left(-\left(\frac{x-\beta}{\alpha}\right)^{-\gamma}\right)
$$

and parameters $\alpha=1.928, \beta=-2, \gamma=10$. Thereby, the normal distribution and the Fréchet distribution satisfy $\operatorname{med}\left(E_{n}\right)=0$, but only the Fréchet distribution also satisfies the conditions of Theorem 4.1 for consistency, if the starting value is large enough. But the aim of the simulation study is to show how the proposed simplicial depth test behaves if the assumptions are not completely satisfied. In particular, the errors with contaminated normal distribution provide innovation outliers in the sense of Fox (1972).

Figure 3 shows the power functions for the normally distributed errors. Thereby, the horizontal and vertical lines denote the components of $\theta^{0}$ so that their intersection is $\theta^{0}$. One can clearly see, that the OLS test performs best under the normal distribution. This is not surprising, since it assumes the correct error distribution. The sign test behaves quite well close to the alternative. Unfortunately in case of explosive processes the power also decreases when $\theta_{0}$ and $\theta_{1}$ lead to residuals which have a poor fit but indicate an error median of zero. This for example happens, if the first half of residuals is positive and the second half is negative. As a result this test is very unstable in case of explosive $\mathrm{AR}(1)$ processes. The $d_{S}$ test clearly outperforms the simplified depth tests. It also shows a better performance than the OLS test in direction of a diagonal with positive slope, but accepts a wider range of values on a diagonal with negative slope.

In Figure 4, the comparison for errors with the contaminated normal distribution is depicted, and Figure 5 provides the comparison for errors with the Fréchet distribution. Now the simplicial depth test performs clearly best. The OLS test suffers from heavy bias due to the skewed error distributions and the sign test still shows the identifiability problem.

In Figures 6, 7, 8, we compare the tests evaluated on the diagonal given by $\theta_{0}=50.7-50 \cdot \theta_{1}$, where the slope of the diagonal is negative. The straight line goes from $(-0.325,1.0205)$ to $(0.725,0.9995)$ through $H_{0}$ defined by $\theta=(0.2,1.01)^{T}$. In the Figures the $\mathrm{x}$-axis is defined by the parameter $\lambda \in[0,1]$ from the parametric form of the straight line given by $(0.725,0.9995)^{T}+\lambda \cdot(-1.05,0.021)^{T}$. On this line $\lambda=0.5$ coincides with $H_{0}$. Here, the main advantage of the full simplicial depth compared to the sign test is clearly visible. Additionally, these figures show how the new test outperforms the OLS test in the case of nonnormal errors where the OLS test in particular does not keep the level anymore. 
Summarising we see, that the $d_{S}$ test can be applied to explosive AR(1) processes under quite general conditions and does not suffer of systematic failure or heavy bias in case of skewed errors or outliers. Further, by the price of additional computational costs, the full simplicial depth statistic defines a test with higher power than the simplified statistics based on simplicial depth.

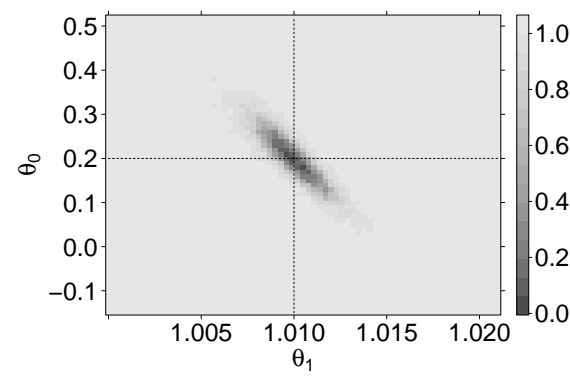

(a) $d_{S}$

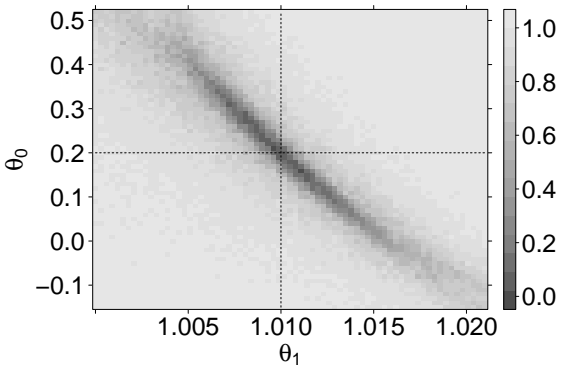

(c) $d_{S}^{2}$

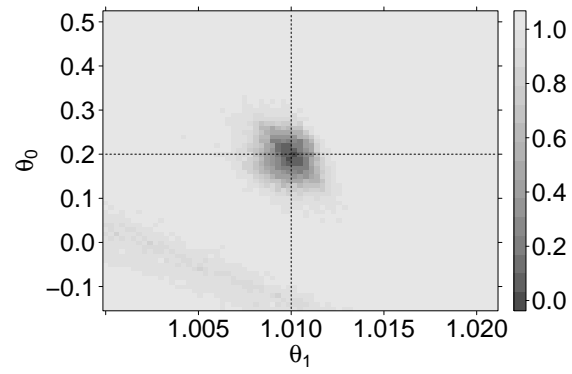

(e) OLS

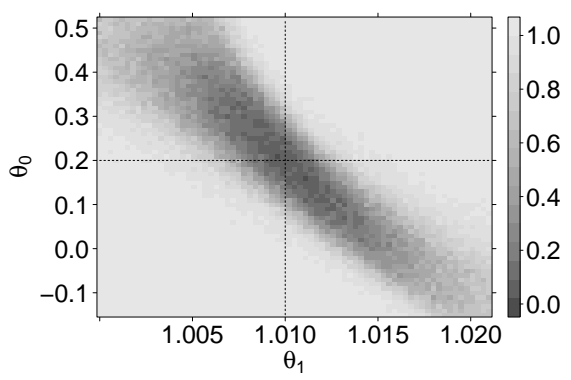

(b) $d_{S}^{1}$

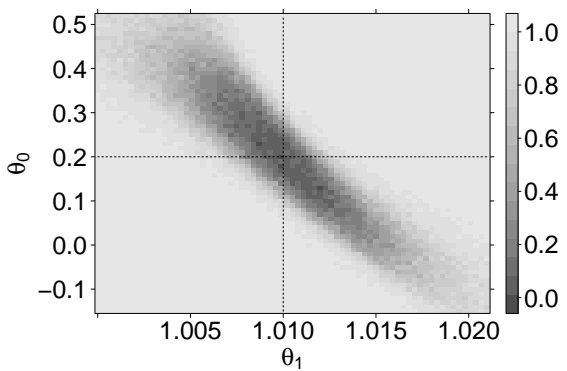

(d) $d_{S}^{3}$

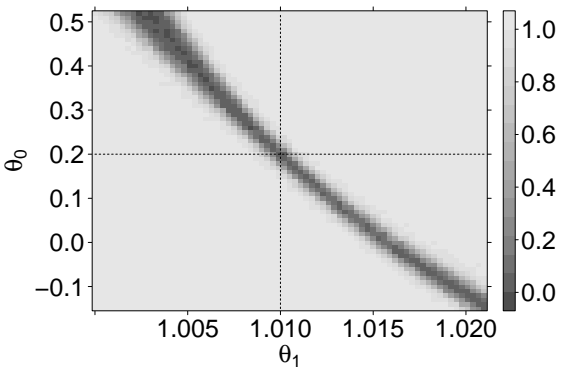

(f) Sign

FiguRE 3. Power of the tests based on normally distributed errors 


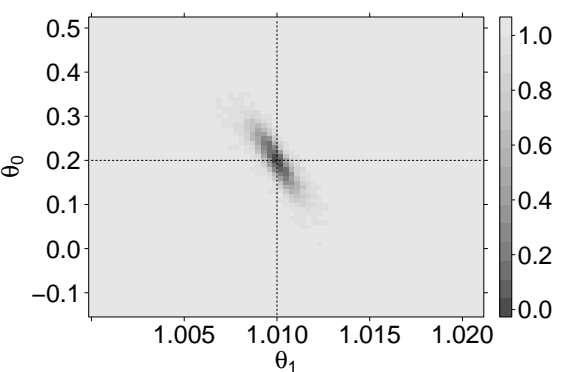

(a) $d_{S}$

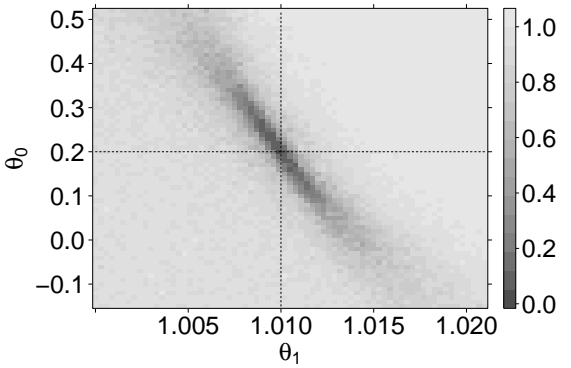

(c) $d_{S}^{2}$

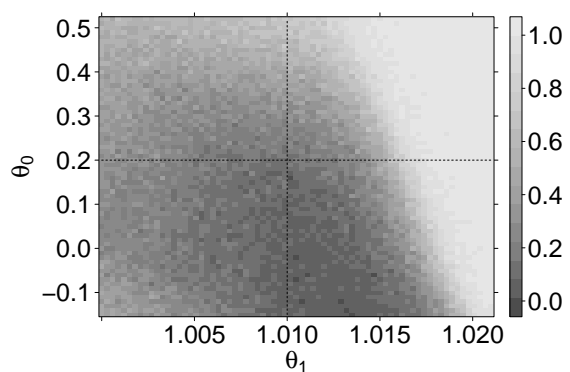

(e) OLS

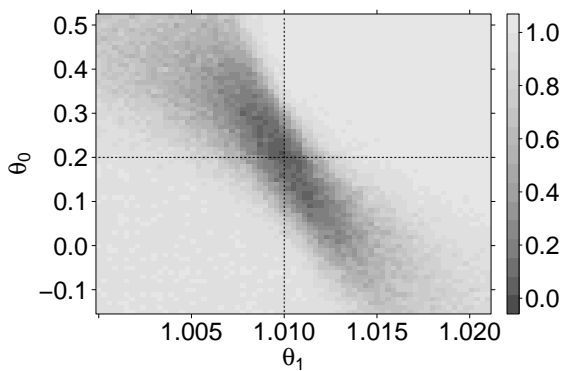

(b) $d_{S}^{1}$

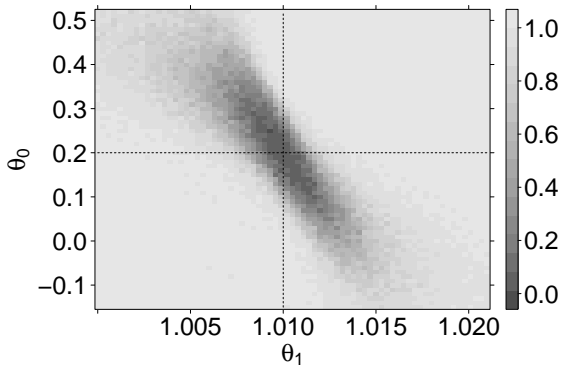

(d) $d_{S}^{3}$

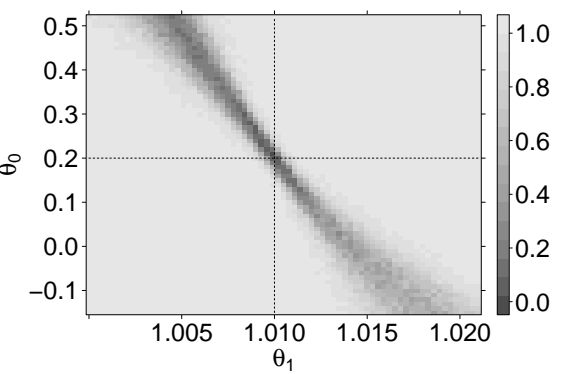

(f) Sign

Figure 4. Power of the tests based on errors with contaminated normal distribution 


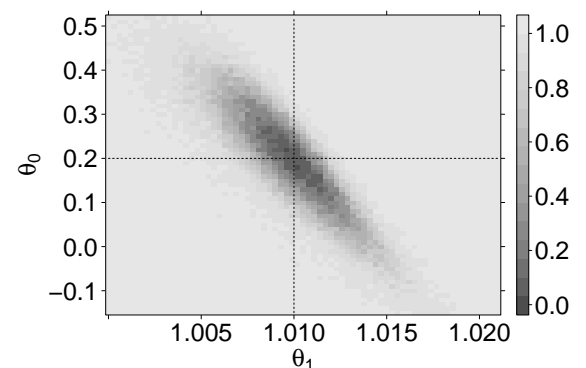

(a) $d_{S}$

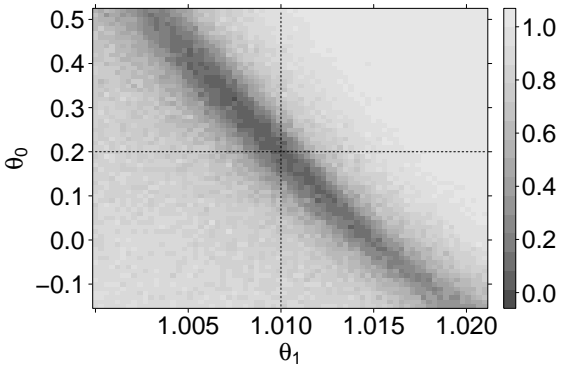

(c) $d_{S}^{2}$

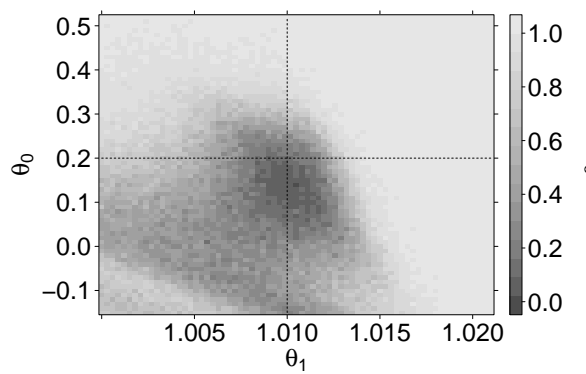

(e) OLS

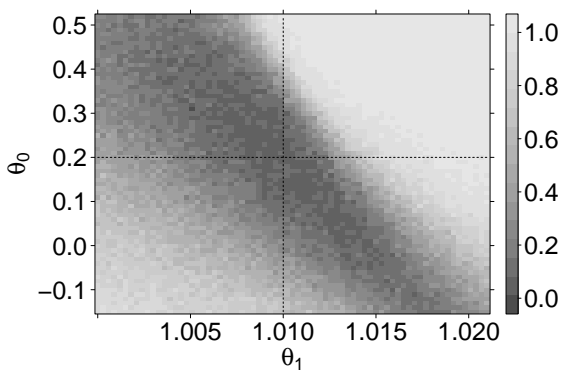

(b) $d_{S}^{1}$

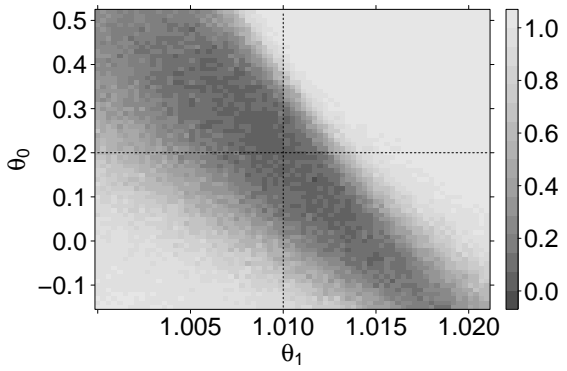

(d) $d_{S}^{3}$

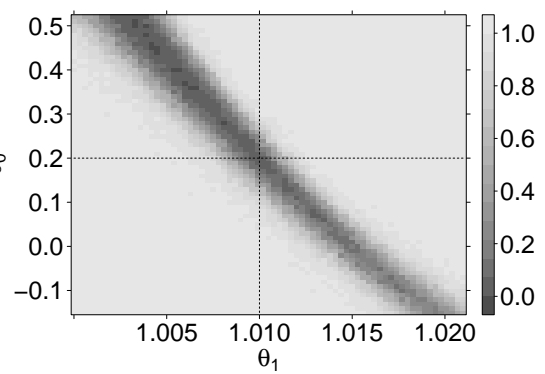

(f) Sign

FiguRE 5. Power of the tests based on errors with Fréchet distribution 


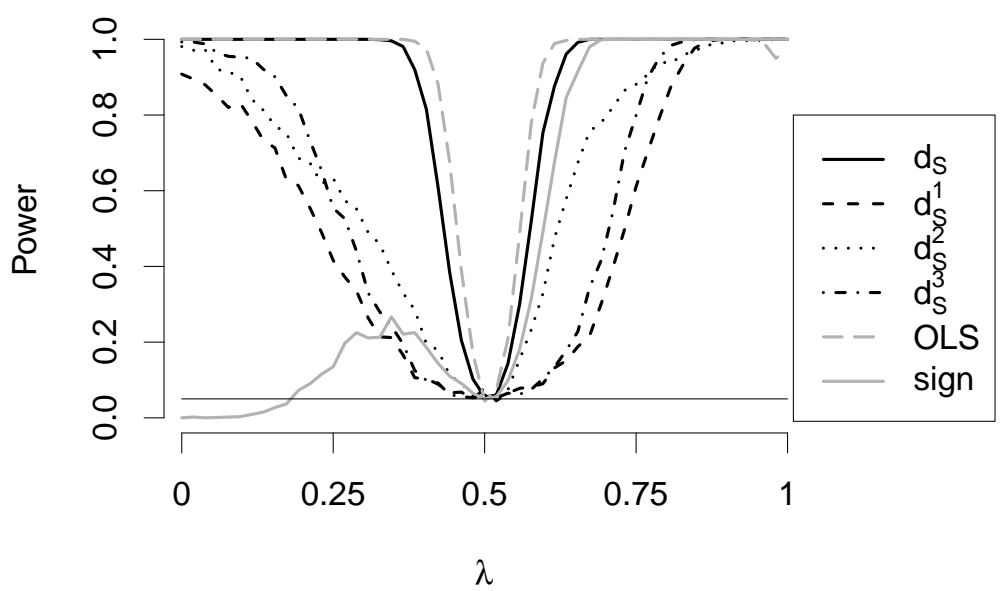

Figure 6. Power evaluated along $\theta=(0.725,0.9995)^{\top}+$ $\lambda(-1.05,0.021)^{\top}$ for normally distributed errors

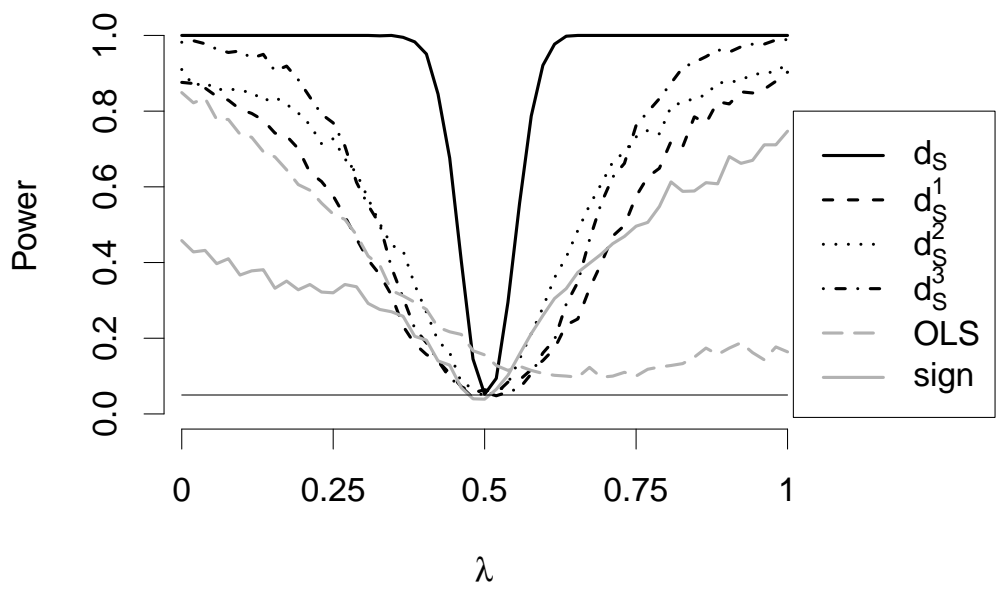

Figure 7. Power evaluated along $\theta=(0.725,0.9995)^{\top}+$ $\lambda(-1.05,0.021)^{\top}$ for errors with contaminated normal distribution 


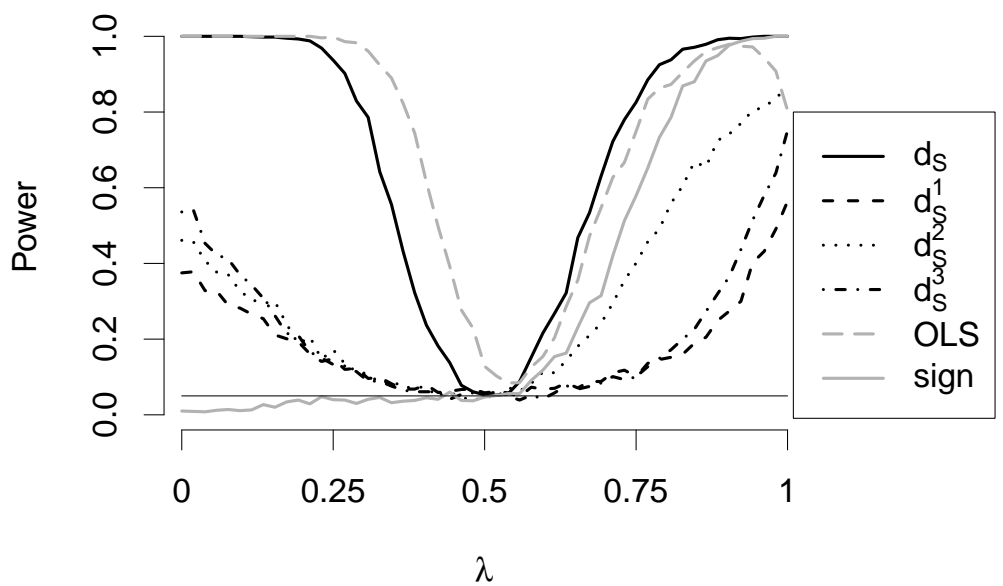

Figure 8. Power evaluated along $\theta=(0.725,0.9995)^{\top}+$ $\lambda(-1.05,0.021)^{\top}$ for errors with Fréchet distribution 


\section{REFERENCES}

Agostinelli, C. (2003). Robust time series estimation via weighted likelihood. In Developments in Robust Statistics, eds. R. Dutter, P. Filzmoser, U. Gather, P. J. Rousseeuw, Physica-Verlag, Heidelberg, 266-276.

Anderson, T. (1959). On asymptotic distributions of estimates of parameters of stochastic difference equations. Ann. Math. Statist. 30, 676-687.

Basawa, I., Mallik, A., McCormick, W., Taylor, R. (1989). Bootstrapping explosive autoregressive processes. Ann. Statist. 17, 1479-1486.

Bazarova, A., Berkes, I. and Horváth, L. (2014). Trimmed stable AR(1) processes. To appear in Stochastic Process. Appl.

Boldin, M. V. (2011). Local robustness of sign tests in AR(1) against outliers. Math. Methods Statist. 20(1), 1-13.

Denecke, L., and Müller, Ch.H. (2011). Robust estimators and tests for bivariate copulas based on likelihood depth. Comput. Statist. Data Anal 55, 2724-2738.

Denecke, L., and Müller, Ch.H. (2012). Consistency and robustness of tests and estimators based on depth. J. Statist. Plann. Inference 142, 2501-2517.

Denecke, L., and Müller, Ch.H. (2013). New robust tests for the parameters of the Weibull distribution for complete and censored data. To appear in Metrika. DOI:10.1007/s00184-013-0454-8.

Denecke, L., and Müller, Ch.H. (2014). Consistency of the likelihood depth estimator for the correlation coefficient. Statist. Papers 55, 3-13.

Fox, J. (1972). Outliers in time series. J. Roy. Statist. Soc. 43, 350-363.

Fried, R. und Gather, U. (2005). Robust trend estimation for AR(1) disturbances. Austrian J. Statist. 34, 139-151.

Gelper, S., Fried, R., Croux, C. (2009). Robust forecasting with exponential and Holt-Winters smoothing. J. Forecast. 29, 285-300.

Grillenzoni, C. (2009). Robust non-parametric smoothing of non-stationary time series. J. Stat. Comput. Simul. 79, 379-393.

Grossi, L. und Riani, M. (2002). Robust time series analysis through the forward search. In Proc. Comput. Statist., eds. W. Härdle, B. Rönz, Physica-Verlag, Heidelberg, 521-526.

Huggins, R. (1989). The sign test for stochastic processes. Aust. N. Z. J. Stat. 31, 153-165.

Hwang, S.Y. (2013). Arbitrary initial values and random norm for explosive AR (1) processes generated by stationary errors. Statist. Probab. Lett., 83, 127-134.

Hwang, S.Y. and Basawa, I.V. (2005). Explosive random coefficient AR(1) processes and related asymptotics for least squares estimation. J. Time Series Anal. 26, 807824.

Hwang, S.Y., Kim, S., Lee, S.D., Basawa, I.V. (2007). Generalized least squares estimation for explosive AR(1) processes with conditionally heteroscedastic errors. Statist. Probab. Lett. 77, 1439-1448.

Liu, R.Y. (1988). On a notion of simplicial depth. Proc. Nat. Acad. Sci. USA 85, $1732-1734$.

Liu, R.Y. (1990). On a notion of data depth based on random simplices. Ann. Statist. 18, 405-414.

Kustosz, C. P. and Müller, Ch. H. (2014a). Analysis of crack growth with robust distribution-free estimators and tests for nonstationary autoregressive processes. Statist. Papers 55, 125-140. 
Kustosz, C. P. and Müller, Ch. H. (2014b). Simplified simplicial depth for regression and autoregressive growth processes. Submitted.

Maronna, R. (2006). Robust Statistics: Theory and Methods. Wiley, New York.

Mizera, I. (2002). On depth and deep points: a calculus. Ann. Statist. 30, 1681-1736.

Müller, Ch.H. (2005). Depth estimators and tests based on the likelihood principle with application to regression. J. Multivariate Anal. 95, 153-181.

Novomestky, F. (2012). matrixcalc: collection of functions for matrix calculations. R package version 1.0-3. http://CRAN.R-project.org/package=matrixcalc

Paulaauskas, V. and Rachev, T. (2003). Maximum likelihood estimators in regression models with infinite variance innovations. Statist. Papers 44, 47-65.

Pollard, D. (1984). Convergence of Stochastic Processes. Springer, New York.

$\mathrm{R}$ Core Team (2013). R: A language and environment for statistical computing. $\mathrm{R}$ Foundation for Statistical Computing, Vienna, Austria. URL http://www.Rproject.org/.

Rousseeuw, P.J. and Hubert, M. (1999). Regression depth. J. Amer. Statist. Assoc 94, 388-433.

Stute, W. and Gründer, B. (1993). Nonparametric prediction intervals for explosive AR(1)-processes. J. Nonparametr. Stat. 2, 155-167.

Tierney, L., Rossini, A.J., Li, N. and Sevcikova, H. (2013). snow: simple network of workstations. $\mathrm{R}$ package version 0.3-13. http://CRAN.Rproject.org $/$ package $=$ snow

Tukey, J.W. (1975). Mathematics and the picturing of data. In Proc. Int. Congress Math., Vancouver 1974, 2, 523- 531.

Van der Vaart, A. W. and Wellner, J. A. (2000). Weak Convergence and Empirical Processes. With Applications to Statistics. Springer, New York.

Venables, W. N. and Ripley, B. D. (2002). Modern Applied Statistics with S. Fourth Edition. Springer, New York.

Wang, X. and Yu, J. (2013). Limit theory for an explosive autoregressive process. Working Paper, No. 08-2013. Singapore Management University, School of Economics.

Wellmann, R., Harmand, P., Müller, Ch.H. (2009). Distribution-free tests for polynomial regression based on simplicial depth. J. Multivariate Anal. 100, 622-635.

Wellmann, R. and Müller, Ch.H. (2010a). Tests for multiple regression based on simplicial depth. J. Multivariate Anal. 101, 824-838.

Wellmann, R. and Müller, Ch.H. (2010b). Depth notions for orthogonal regression. J. Multivariate Anal. 101, 2358-2371.

Yu, H. (2002). Rmpi: parallel statistical computing in R. R News 2/2:10-14. 


